\title{
Steady axial electric field may lead to controllable cross-stream migration of droplets in confined oscillatory microflows
}

\author{
Somnath Santra ${ }^{1}$ and Suman Chakraborty ${ }^{1} \dagger$ \\ ${ }^{1}$ Department of Mechanical Engineering, Indian Institute of Technology Kharagpur, \\ Kharagpur, West Bengal 721302, India
}

(Received 21 January 2020; revised 15 August 2020; accepted 12 September 2020)

Cross-stream migration of a droplet in an incipient flow turns out to be of outstanding importance in several emerging applications encompassing chemistry, engineering and biology. Here, we bring out the confluence of confinement, oscillatory axial pressure gradient and steady axial electric field towards controlling spatiotemporal characteristics of cross-stream migration of droplets in a micro-confined fluidic environment, bearing immense implications in in vitro modelling of bio-analytical procedures. Under the sole influence of an oscillatory axial pressure gradient, the time taken by a droplet to achieve a steady-state transverse position is significantly long and the direction of the droplet's motion cannot be altered at will. However, confinement-modulated electrohydrodynamic interactions enable overcoming this constraint, even when the applied electric field is orthogonal to the intended direction of droplet migration, a proposition that is not feasible in an unbounded domain. Our results reveal that depending on the relative electrical properties of the droplet and the carrier phases and a competing influence of electrical, viscous and capillary stresses, the rate of transverse migration can be controlled by effectively modulating the axial oscillations in its cross-stream motion. Beyond a threshold value of the applied electric field, simultaneous enhancement in the droplet migration rate and reversal in the direction of its lateral migration become possible, which cannot otherwise be achieved by the oscillatory pressure field alone. Furthermore, the oscillatory characteristics in the droplet migration can be dampened out completely by exploiting the addressed physical interplay. Results from in-house experiments corroborate our theoretical conjecture.

Key words: drops and bubbles

\section{Introduction}

Precise manipulation and sorting of soft entities in microfluidic systems have received remarkable attention in contemporary research, as attributable to their diverse applications in physical, biological and engineering systems (Sibillo et al. 2006; Seč et al. 2012;

$\dagger$ Email address for correspondence: suman@mech.iitkgp.ac.in 
Dangla, Kayi \& Baroud 2013; Wioland et al. 2013; Huerre et al. 2015). These perspectives often include material transport and rapid as well as efficient mixing in a technologically advanced platform, in vitro diagnostics, drug discovery and targeted drug delivery (Schwabe et al. 1992; Stone, Stroock \& Ajdari 2004; Teh et al. 2008; Capretto et al. 2011; Casadevall i Solvas \& DeMello 2011). In many of these applications, the fundamental scientific premise that stands out as crucial is an assessment of the cross-stream motion of soft entities (such as droplets, vesicles and cells) in a confined fluidic environment.

Several reported studies pointed out that the migration characteristic of a droplet can be modulated by altering the deformability of the interface (Goldsmith \& Mason 1962; Chaffey, Brenner \& Mason 1965; Haber \& Hetsroni 1971; Wohl \& Rubinow 1974; Stan et al. 2011; Mandal et al. 2015a), fluid properties (Chan \& Leal 1979; Mukherjee \& Sarkar 2013, 2014; Hazra, Mitra \& Sen 2019), flow inertia (Ho \& Leal 1974; Mortazavi \& Tryggvason 2000; Chen et al. 2014) and the nature of flow (steady or oscillatory)(Graham \& Higdon 2000a, 2002; Chaudhury, Mandal \& Chakraborty 2016). In addition to these factors, mutual interactions between electric forcing and domain confinement can also be used as a means of fine-tuning the modulation of the droplet's motion (Deshmukh \& Thaokar 2012; Esmaeeli 2016; Zhang et al. 2016; Brosseau \& Vlahovska 2017; Nath et al. 2018; Santra, Mandal \& Chakraborty 2018b, 2019a; Poddar et al. 2019a).

In the presence of a steady background flow (plane Poiseuille, extensional, simple shear, etc.), numerous studies have been conducted on the motion and deformation dynamics of the droplet (Chan \& Leal 1977, 1979; Leal 1980; Mortazavi \& Tryggvason 2000; Li \& Pozrikidis 2002; Sessoms et al. 2009; Carlson, Do-Quang \& Amberg 2010; Chung et al. 2010; Afkhami, Leshansky \& Renardy 2011; Mukherjee \& Sarkar 2013). However, the implications of a time-varying flow field on the migration characteristics of a droplet have only been explored to a limited extent (Lovalenti \& Brady 1993; Graham \& Higdon 2000b; Sarkar \& Schowalter 2001a,b). Such time-varying flow pulsations, nevertheless, offer several advantages that may turn out to be of immense benefit in practical microfluidic applications. For instance, this enables efficient manipulation and sorting of rigid and deformable entities at very low Reynolds number, which is otherwise unfeasible in conventional steady-flow microfluidic systems (Mutlu, Edd \& Toner 2018; Asghari et al. 2020). Furthermore, owing to the low particle Reynolds number, the shear stress acting on the suspended entities in the microchannel is minimized, which permits the manipulation of cellular entities under physiologically relevant conditions.

The time-varying flow field in a microfluidic channel often involves a time-dependent imposed pressure gradient that causes the temporal alteration in the hydrodynamic forces. When a droplet is placed in a time-varying flow field, its shape evolves with time, along with a time-dependent inertial response. In a related study, Chaudhury et al. (2016) have analysed the migration characteristics of a droplet in an oscillatory microflow. They have shown that, under a sinusoidal time-variant pressure gradient, the droplet moves to the centreline in a helical pathway. Possibly, the most important finding from their study delineates that the droplet shifts to the centreline without any net axial displacement when the frequency of oscillation is beyond a threshold limit.

Irrespective of the obvious pertinence to physiologically relevant processes, an important limitation of oscillatory-flow-driven droplet manipulation turns out to be the fact that the time taken by the droplet to achieve a steady-state transverse position (termed as the focus time in microfluidic technologies) is significantly large and the direction of the droplet's motion cannot be changed at will. In an effort to overcome these limits, electric-field-induced flow manipulation holds the potential to offer a viable alternative. When an electric field is applied on a leaky dielectric droplet suspended in 
another leaky dielectric medium, the disparity in the electrical properties gives rise to net tangential and normal electric stresses at the interface that create circulatory motion inside and outside of the droplet and leads to its prolate (in direction of electric field) or oblate deformation (perpendicular to the direction of electric field). This phenomenon is classically addressed by Taylor's (1966) 'leaky-dielectric theory'. Later, this theory was further developed by Melcher \& Taylor (1969) and others (Torza, Cox \& Mason 1971; Vizika \& Saville 1992; Saville 1997). Two important assumptions of this model are: the fluids have weak but finite conductivities and the charge relaxation time scale is much shorter than the convective time scale. The former assumption considers the aggregation of free charges in the interface and the latter allows us to decouple the electric field equation from the momentum conservation equation for considerable simplification of the mathematical model. In his pioneering work, Taylor (1966) unravelled that the direction of electric shear-driven circulatory flow inside and outside of the droplet in the sole presence of electric field depends on the relative magnitude of conductivity ratio $(R)$ and permittivity ratio $(S)$ of the leaky dielectric system. To infer the sense of deformation, he further established a discriminating function $\left(\Omega_{T}\right)$ comprising the parameters: viscosity ratio $\lambda=\mu_{i} / \mu_{e}$, conductivity ratio $R=\sigma_{i} / \sigma_{e}$ and permittivity ratio $S=\varepsilon_{i} / \varepsilon_{e}$, where the subscript ' $i$ ' refers to properties of the droplet and the subscript ' $e$ ' refers to the properties of the carrier fluid, respectively; the viscosity, conductivity and the permittivity being denoted by $\mu, \sigma$, and $\epsilon$, respectively. As per Taylor's theory, the discriminating function is defined as $\Omega_{T}=R^{2}+1-2 S+3(R-S)(3 \lambda+2) / 5(\lambda+1)$. Taylor showed that droplet deforms into a prolate (or oblate) configuration for $\Omega_{T}>0$ (or $\Omega_{T}<0$ ). Notably, this theory had been essentially premised on the consideration of an unbounded domain.

Motivated by the classical work of Taylor (1966), several studies have been reported on electrically modulated morpho-dynamics of droplets in an external flow (Hase, Watanabe \& Yoshikawa 2006; Ristenpart et al. 2009; Mhatre \& Thaokar 2013; Zhang et al. 2016; Brosseau \& Vlahovska 2017; Poddar et al. 2018, 2019b; Behera et al. 2019; Santra et al. 2018b, 2019a; Santra, Das \& Chakraborty 2020). Mandal, Bandopadhyay \& Chakraborty (2016) studied the cross-stream migration of the droplet in unbounded plane Poiseuille flow under uniform electric field. They have shown that the droplet can migrate toward the centreline or wall electrode, depending on the relative electrical parameters. However, one important conclusion drawn from their study is that the cross-stream motion is possible only when the electric field makes an angle with the flow direction. Hence, an axial electric field has no effect on the droplet's cross-stream motion in an unbounded domain. In a recent study, Santra et al. (2018b) have introduced the effect of domain confinement in electrohydrodynamics of droplets and obtained that the essential conditions of oblate and prolate deformation become reversed below a critical relative dimension of the domain confinement. This study has opened up the possibility of altering the established features of electrohydrodynamic manipulation of droplets in unbounded domains via confinement-mediated interactions.

Here, we unveil the migration characteristics of a leaky dielectric droplet under the combined confluence of a steady axial electric field, domain confinement and oscillatory pressure-driven flow, from both computational and experimental perspectives. In sharp contrast to reported theory (Mandal et al. 2016) that depicts the possibility of cross-stream migration of a droplet only if subjected to a tilted electric field, we show that confinement-induced electrohydrodynamic interactions enable the spatiotemporal characteristics of lateral motion of a droplet to be controlled even in the presence of an electrical field that is orthogonal to the direction of the droplet migration. In addition, we offer insights on controlling and stabilizing the oscillatory characteristics of transverse migration of the droplet before its eventual settling, along with a simultaneous reversal 
(a)

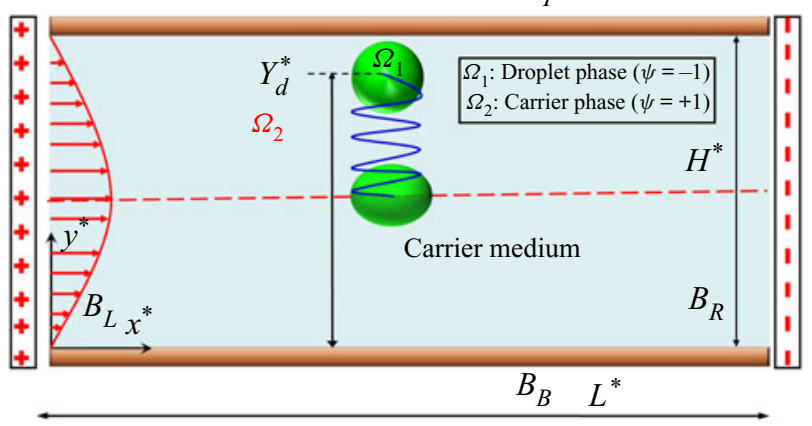

(b)

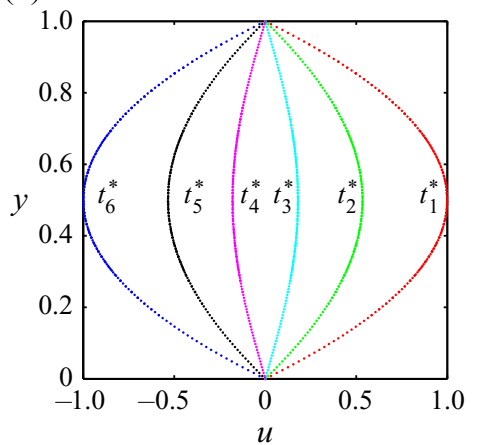

FIgURE 1. (a) Schematic of the rectangular computational domain. The length and width are $L^{*}$ and $H^{*}$, respectively. The numerical set-up is bounded by the top and bottom bounding walls $\left(B_{T}, B_{B}\right)$ and the left and right boundaries $\left(B_{L}, B_{R}\right)$. The initial distance of the droplet from the lower electrode is denoted by the $Y_{d}^{*}$. (b) Time sequence of the velocity profile, where $t_{6}^{*}>t_{5}^{*}>$ $t_{4}^{*}>t_{3}^{*}>t_{2}^{*}>t_{1}^{*}$.

in its direction of migration, which has not been addressed earlier. Our results further illustrate the establishment of an explicit control over the time taken by the droplet to achieve a steady-state transverse position, under the combined influence of confined oscillatory hydrodynamics and axial electrical forcing. Experimental investigations support these essential findings. Results from the present study may find important applications (Dey, Chakraborty \& Chakraborty 2011; Goswami \& Chakraborty 2011; Bakli \& Chakraborty 2012; Bandopadhyay \& Chakraborty 2012a,b; Yavari et al. 2012; Rana et al. 2014) in rapid and controlled focusing of soft and deformable entities at will, in various physical, chemical and biological processes having inherent limitations associated with their finite length and focus time.

\section{Numerical methodology}

The two-dimensional (2-D) computational domain is shown in figure 1, where a neutrally buoyant leaky dielectric droplet is suspended in another leaky dielectric medium under the combined governance of oscillatory pressure-driven background flow and uniform axial electric field. As the computations are planar (2-D), the undeformed droplet is circular in shape. The computational domain is bounded by the top wall boundary $\left(B_{T}\right)$, bottom wall boundary $\left(B_{B}\right)$, inlet left-side boundary $\left(B_{L}\right)$ and the outlet right-side boundary $\left(B_{R}\right)$. The radius of the droplet is denoted by $a$. The initial location of the droplet from the bottom wall boundary is $Y_{d}^{*}$. The electrodes are located at the side boundaries $\left(B_{L}\right.$ and $\left.B_{R}\right)$. Thus, an axial electric field, $E^{*}$ acts on the droplet. In this study, a Cartesian coordinate system is adopted, which is fixed at the bottom wall boundary, as depicted in figure 1 .

\subsection{Numerical simulation: phase field method}

For computational modelling of the physical problem described previously, we adapt the phase field method (Jacqmin 1999; Badalassi, Ceniceros \& Banerjee 2003; Mandal et al. 2015b). In previously reported studies (Wang, Qian \& Sheng 2008; Mandal et al. 2015b; Chaudhury et al. 2016), several authors mentioned the utility of this method in capturing the interfacial dynamics of a two-fluid system. It is important to mention that 
the method is premised on the minimization of the total energy of the system and is thermodynamically consistent (Chakraborty 2007, 2008). Owing to its flexible generalized framework, it allows the incorporation of appropriate physics by suitably altering the free energy functional. In the phase field method, for identifying the distribution of constituting fluid phases, an order parameter, $\psi(x, t)$ is used. In figure 1, the droplet and surrounding fluid medium are denoted by $\psi=-1$ and +1 , respectively. The values of $\psi$ vary between -1 and 1 in the diffuse interfacial region. The dynamic evolution of $\psi$ is described by the Cahn-Hilliard equation. This equation comprises both the advection as well as diffusion terms and reads (Jacqmin 1999; Mandal et al. 2015b)

$$
\frac{\partial \psi}{\partial t^{*}}+u^{*} \cdot \nabla^{*} \psi=\nabla^{*} \cdot\left(M_{\psi}^{*} \nabla^{*} G^{*}\right)
$$

where the mobility factor and the chemical potential are denoted by $M_{\psi}^{*}, G^{*}$, respectively. Here $G^{*}$ is described as $G^{*}=\gamma\left(\psi^{3}-\psi\right) / \xi^{*}-\gamma \xi^{*} \nabla^{* 2} \psi$. The interfacial thickness is denoted by $\xi^{*}$. In this work, asterisks are used to denote dimensional parameters, for notational convenience.

In the framework of phase field formalism, any generic fluid property $\left(\chi^{*}\right)$ can be suitably interpolated via the distribution of the order parameter (Badalassi et al. 2003; Yang, Li \& Ding 2013; Mandal et al. 2015b; Yang et al. 2016):

$$
\chi^{*}=\frac{(1-\psi)}{2} \chi_{i}^{*}+\frac{(1+\psi)}{2} \chi_{e}^{*}
$$

In a non-dimensional format, this is expressed as

$$
\chi=\frac{(1-\psi)}{2} \chi_{r}+\frac{(1+\psi)}{2}, \quad \text { where } \chi_{r}=\chi_{i}^{*} / \chi_{e}^{*}
$$

\subsection{Governing equations and boundary conditions}

\subsubsection{Electric field}

As the electric field is irrotational $\left(\nabla^{*} \times E^{*}=0\right)$, it may be related to the electric potential as $E^{*}=-\nabla^{*} \phi$. Forces of electrical origin may be expressed in terms of the divergence of the Maxwell stress tensor, so that

$$
\boldsymbol{F}^{* E}=\int_{\forall}\left(\nabla^{*} \cdot \boldsymbol{T}^{* M}\right) \mathrm{d} x^{* 3}, \quad \nabla^{*} \cdot \boldsymbol{T}^{* M}=q_{v}^{*} \boldsymbol{E}^{*}-\frac{1}{2}\left(\boldsymbol{E}^{*} \cdot \boldsymbol{E}^{*}\right) \cdot \nabla^{*} \varepsilon,
$$

where $\forall$ symbolizes the domain volume, and the Maxwell stress tensor is denoted by $T^{* M}$. The first term of (2.4) denotes the Coulomb force (or electric force), which appears because of the interaction between the electric field and free charges. The second term refers to dielectrophoretic force. In (2.4), $q_{v}$ denotes the bulk-free charge density. In accordance with Gauss law, one may write

$$
\nabla^{*} \cdot\left(\varepsilon \nabla^{*} \phi\right)=q_{v}^{*}
$$

Following the generic property interpolation scheme described earlier, $\epsilon$ can be described as

$$
\varepsilon=\frac{(1-\psi)}{2} \varepsilon_{i}+\frac{(1+\psi)}{2} \varepsilon_{e}
$$


Further, from charge conservation consideration, the bulk-free charge density follows the following governing equation:

$$
\frac{\mathrm{D} q_{v}^{*}}{\mathrm{D} t^{*}}+\nabla^{*} \cdot\left(\sigma \boldsymbol{E}^{*}\right)=\frac{\partial q_{v}^{*}}{\partial t^{*}}+\nabla^{*} \cdot\left(q_{v}^{*} \boldsymbol{u}^{*}\right)+\nabla^{*} \cdot\left(\sigma \boldsymbol{E}^{*}\right)=0 .
$$

In accordance with the leaky-dielectric theory (Taylor 1966; Melcher \& Taylor 1969), free charges are transferred instantly from bulk fluid to the interfacial region and the bulk fluid becomes free of charge. Hence, $\mathrm{D} q_{v}^{*} / \mathrm{D} t^{*}=0$ and (2.7) is reduced to the following form:

$$
\nabla^{*} \cdot\left(\sigma E^{*}\right)=0 .
$$

Using the irrotationality of the electric field, (2.8) can be expressed as

$$
\nabla^{*} \cdot\left(\sigma \nabla^{*} \phi\right)=0 .
$$

Here $\sigma$ is interpolated as $\sigma=((1-\psi) / 2) \sigma_{i}+((1+\psi) / 2) \sigma_{e}$.

At the inlet and outlet boundaries, the electric potential follows the boundary conditions as stated in the following:

$$
\left.\begin{array}{l}
\text { At inlet boundary }\left(B_{L}\right): \phi^{*}=0 \text { at } x^{*}=0, \\
\text { At outlet boundary }\left(B_{R}\right): \phi^{*}=L^{*} E_{\infty}^{*} \text { at } x^{*}=L^{*} .
\end{array}\right\}
$$

\subsubsection{Fluid flow}

The governing continuity and momentum equations for fluid flow are given by

$$
\begin{aligned}
& \boldsymbol{\nabla}^{*} \cdot \boldsymbol{u}^{*}=0, \\
\rho\left(\frac{\partial \boldsymbol{u}^{*}}{\partial t^{*}}+\nabla^{*} \cdot\left(\boldsymbol{u}^{*} \boldsymbol{u}^{*}\right)\right)= & -\boldsymbol{\nabla}^{*} p^{*}+\boldsymbol{\nabla}^{*} \cdot\left[\mu\left\{\boldsymbol{\nabla}^{*} \boldsymbol{u}^{*}+\left(\boldsymbol{\nabla}^{*} \boldsymbol{u}^{*}\right)^{\mathrm{T}}\right\}\right] \\
& +G^{*} \boldsymbol{\nabla}^{*} \psi+\boldsymbol{F}^{* E}+F_{o}^{*} \sin \left(\omega^{*} t^{*}\right) \hat{\boldsymbol{e}}_{x} .
\end{aligned}
$$

Equation (2.12) couples the electrohydrodynamics with phase field formalism, where $G^{*} \nabla^{*} \psi$ acts as a representation of the interfacial tension in terms of the phase field order parameter and the chemical potential and $\boldsymbol{F}^{* E}$ denotes the electric body force as stated in (2.4). Here $F_{o}^{*} \sin \left(\omega^{*} t^{*}\right)$ represents the oscillatory component of the driving pressure gradient, where $\omega^{*}$ and $F_{o}^{*}$ represent the frequency and amplitude of the imposed oscillation, respectively.

\subsection{Normalization of governing equations}

Normalized forms of the governing equations, described previously, read

$$
\begin{gathered}
\frac{\partial \psi}{\partial \bar{t}}+\boldsymbol{u} \cdot \boldsymbol{\nabla} \psi=\frac{1}{P e} \nabla^{2} G, \quad \text { where } G=\frac{1}{C n}\left(\psi^{3}-\psi\right)-C n \nabla^{2} \psi, \\
\nabla \cdot(\sigma \nabla \phi)=0, \\
\nabla \cdot \boldsymbol{u}=0, \\
\operatorname{Re}\left(\frac{\partial \boldsymbol{u}}{\partial t}+\boldsymbol{u} \cdot \nabla \boldsymbol{u}\right)=-\nabla p+\nabla \cdot\left[\mu\left\{\nabla \boldsymbol{u}+(\nabla \boldsymbol{u})^{\mathrm{T}}\right\}\right] \\
+\frac{1}{C a} \cdot G \nabla \psi+\frac{C a_{E}}{C a} \boldsymbol{F}^{E}+8 \sin (t S t) \hat{\boldsymbol{e}}_{x} .
\end{gathered}
$$


For the normalization scheme, the following scaling parameters are used: length scale $H^{*}$, velocity scale $u^{*}$, time scale $H^{*} / u_{c}^{*}$, viscous stress scale $\mu_{e} u_{c}^{*} / H^{*}$, electric field scale $E_{\infty}^{*}$ and electric stress scale $\varepsilon_{e} E_{\infty}^{* 2}$. Here $H^{*}$ and $u^{*}$ denote the channel height and centreline flow velocity, respectively. The consequent normalization results in the following dimensionless parameters: Reynolds number $\left(\operatorname{Re}=\rho u_{c}^{*} H^{*} / \mu_{e}\right)$, Péclet number $\left(P e=H^{* 2} u_{c} / M_{\psi}^{*} \gamma\right)$, capillary number $\left(C a=\mu u_{c}^{*} / \gamma\right)$, electric capillary number $\left(C a_{E}=\right.$ $\left.\varepsilon_{e} E_{\infty}^{* 2} H^{*} / \gamma\right)$, Cahn number $\left(C n=\xi^{*} / H^{*}\right)$ and Strouhal number $\left(S t=\omega^{*} H^{*} / u_{c}^{*}\right)$. Another important dimensionless parameter is the confinement ratio $(W c)$, defined as the ratio of undeformed droplet diameter to the width of the channel (expressed as $W c=2 a^{*} / H^{*}$ ). In the present analysis, we consider $L\left(=L^{*} / H^{*}\right)=3$.

Interpolation of physical properties, in a dimensionless form, reads (Mondal et al. 2014)

$$
\left.\begin{array}{c}
\rho=\frac{(1-\psi)}{2} \rho_{r}+\frac{(1+\psi)}{2} ; \rho_{r}=\frac{\rho_{i}}{\rho_{e}} \\
\mu=\frac{(1-\psi)}{2} \lambda+\frac{(1+\psi)}{2} ; \lambda=\frac{\mu_{i}}{\mu_{e}} \\
\varepsilon=\frac{(1-\psi)}{2} S+\frac{(1+\psi)}{2} ; S=\frac{\varepsilon_{i}}{\varepsilon_{e}} \\
\sigma=\frac{(1-\psi)}{2} R+\frac{(1+\psi)}{2} ; R=\frac{\sigma_{i}}{\sigma_{e}}
\end{array}\right\}
$$

As the interacting fluids are neutrally buoyant for the case studies addressed in this work, we have set $\rho=\rho_{r}=1$ subsequently.

The boundary conditions employed at the top and bottom walls $\left(B_{T}\right.$ and $\left.B_{B}\right)$ in dimensionless forms read

(i) No slip : $\boldsymbol{u}-\left(\boldsymbol{u} \cdot \boldsymbol{n}_{s}\right) \boldsymbol{n}_{s}=\mathbf{0}$,

(ii) No penetration: $\boldsymbol{u} \cdot \boldsymbol{n}_{s}=0$,

(iii) No flux : $\boldsymbol{n}_{s} \cdot \nabla \psi=0$.

Here, $\boldsymbol{n}_{s}$ denotes the normal vector at the walls. The governing equations have been solved using the finite element method taking the mentioned boundary conditions into consideration (Mandal et al. 2015b; Santra et al. 2019b; Wang et al. 2019). The details of the numerical implementation are described in the supplementary material available at https://doi.org/10.1017/jfm.2020.789.

\section{Results and discussion}

\subsection{Model benchmarking}

In an effort to benchmark our numerical model, we have first conducted validation studies vis-à-vis the results reported by Halim \& Esmaeeli (2013), Mortazavi \& Tryggvason (2000), Chaudhury et al. (2016) and Ha \& Yang (2000). Ha \& Yang (2000) experimentally investigated the electric-field-induced deformation and breakup characteristics of Newtonian and non-Newtonian droplets.

For comparison, we have chosen system NN21 from their study, where castor oil and silicone oil $\left(\mu^{*}=0.90 \mathrm{~Pa} \cdot \mathrm{s}\right)$ have been used as the droplet phase and suspending fluid phase, respectively. In the sole presence of an electric field, the droplet either deforms 
into prolate or oblate configuration depending on the relative electrical properties. The degree of the deformation of the droplet is measured by the deformation parameter, $D$ (Taylor 1966), expressed as $D=\left(L_{\max }-L_{\min }\right) /\left(L_{\max }+L_{\min }\right)$, where $L_{\max }$ and $L_{\min }$ are the length of the major axis and minor axis of the elliptically deformed (prolate or oblate) droplet, respectively. In the study reported by Halim \& Esmaeeli (2013), they have employed the front-tracking/finite difference method to study the transient electrohydrodynamic behaviour of leaky dielectric droplet under uniform electric field. In the numerical study performed by Chaudhury et al. (2016), they have analysed the migration characteristic of the droplet in the presence of background oscillatory flow field in a parallel plate microchannel. Mortazavi \& Tryggvason (2000) have performed a numerical study on the cross-stream motion of the deformable droplet in pressure-driven flow, taking the effect of finite inertia, the viscosity ratio of the fluids and the interfacial tension into consideration. In this study, they have used a front-tracking/finite difference method to numerically simulate the problem. Figure 2(a) depicts an excellent agreement between the findings of Halim \& Esmaeeli (2013) and predictions based on the present numerical set-up, highlighting the temporal variation of the electric field-induced deformation of droplet for different values of the permittivity ratio. Figure $2(b)$ also presents another comparison of the cross-stream motion of the droplet between the present findings and the numerical results of Mortazavi \& Tryggvason (2000), exhibiting excellent quantitative agreement. In addition, figure 2(c) illustrates a comparison between the present numerical results and experimental results of Ha \& Yang (2000) on the electric-field-induced alteration of the steady-state deformation of the droplet, illustrating favourable agreement. Figure $2(d)$ depicts a comparison between our numerical results and the results of Chaudhury et al. (2016) on the cross-stream migration of the droplet under an oscillatory flow field. Excellent agreement is also obtained in this regard. All these benchmarking studies also involve a rigorous grid independence (equivalently, Cahn number independence) study and Péclet number independence test, which have not been detailed in this work for the sake of brevity. These validations enable benchmarking optimal grid sizing in the computational domain, based on the consideration of Cahn number independence (equivalently, grid independence). The details of the Cahn number independence and grid independence studies are given in the supplementary material.

\subsection{Alteration in droplet motion in the combined presence of an electric field and background oscillatory flow}

\subsubsection{Electric-field-induced modification in cross-stream motion of the droplet}

Figure 3 depicts the influence of a steady axial electric field on the cross-stream motion of the droplet for a model system based on the problem description outlined schematically in figure 1. Values of the relevant dimensionless parameters (unless they are varied) used for generating these results are $(S, R)=(2,0.5), \rho_{r}=1$ and $\lambda=1$. These collective properties conform to a specific category of physical system designated as system A. In the present analysis, depending on the relative strength of electric stress as compared with the flow-induced viscous stress, we have classified the magnitude of electric field strength into three categories: (i) low electric field strength (where $C a_{E} \leq 0.5$ and $\mathrm{Ca}=0.3$ ); (ii) moderate electric field strength $\left(0.5<C a_{E} \leq 1.5\right.$ and $\left.C a=0.3\right)$; (iii) high electric field strength $\left(C a_{E}>1.5\right.$ and $\left.C a=0.3\right)$.

The shape evolution of the droplet at different stages of the cycle of the imposed oscillation is also shown in figure 3. In the sole presence of oscillatory pressure-driven flow, the droplet moves towards the centreline in a zig-zag pathway without any net axial 

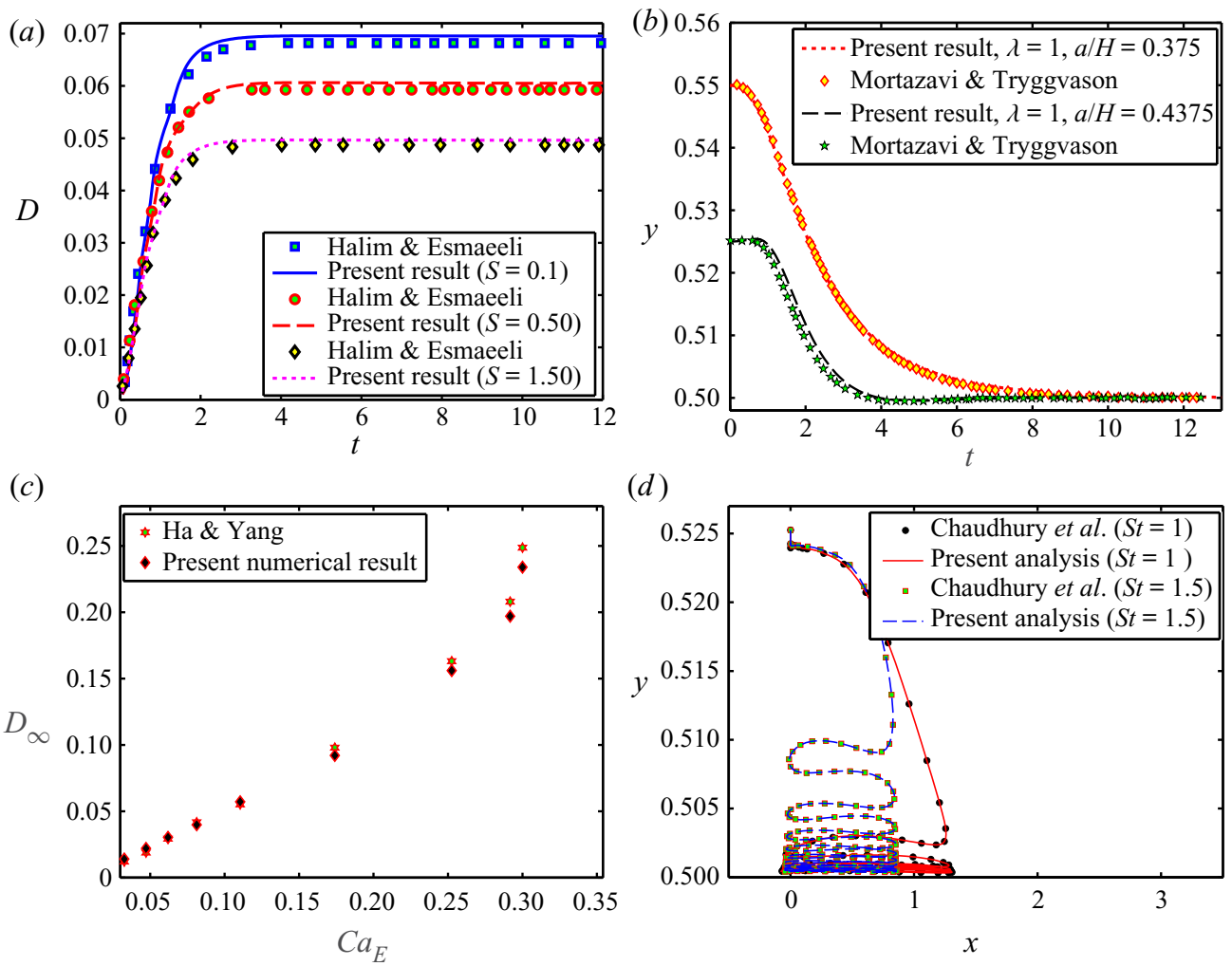

FIGURE 2. (a) Temporal variation of the deformation parameter in the presence of a uniform electric field, considering the set-up of Halim \& Esmaeeli (2013). Important parameters are $R=2.5, \lambda=0.1, C a_{E}=0.25$ and $R e=1$. (b) Temporal variation of the transverse position of the droplet's centroid, considering the set-up of Mortazavi \& Tryggvason (2000). Important parameters are $\rho_{r}=1, R e=1$ and $C a=0.33$. (c) Variation of steady-state parameters $\left(D_{\infty}\right)$ with electric field strength $\left(\mathrm{Ca}_{E}\right)$, considering the set-up of Ha \& Yang (2000). Important parameters are $(R, S)=(10,1.37), R e=0.01$ and $\lambda=0.874$. (d) Cross-stream migration characteristics of the droplet in the presence of oscillatory pressure gradient-driven flow in parallel plate micro-confinement, considering the set-up of Chaudhury et al (2016). Important parameters are $a=0.4375, C a=0.286, \rho_{r}=1, \lambda=1, R e=1$.

displacement (defined as oscillatory motion). However, when an axial electric field is applied, figure 3(a) clearly depicts that the axial oscillations of the droplet prior to reaching the domain centreline dampen out, and the droplet follows an uncurling pathway (defined as non-oscillatory motion) at high values of $\mathrm{Ca}_{E}$. Once the droplet arrives at the centreline, it continues to oscillate in the axial direction. Figure $3(b)$ further emphasizes that the time taken by the droplet to achieve its steady-state transverse position $\left(t_{s s}\right)$ also decreases with the rise in the relative strength of the electric field. Though this phenomenon appears intuitive, it is of fundamental importance in the exploration of several practical problems of outstanding relevance, such as high-throughput and on-demand sorting of deformable entities (droplet, biological cells, etc.) in confined micro-environments. In this regard, it should be mentioned that despite a widespread emergence of oscillatory microfluidics towards sorting soft fluidic and cellular matters under physiologically relevant conditions, one important inherent limitation of the same has been reported to be the fact that the 
(a)

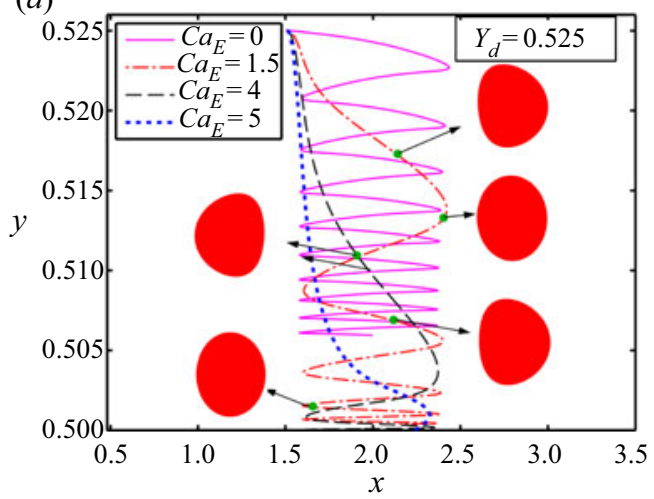

(b)

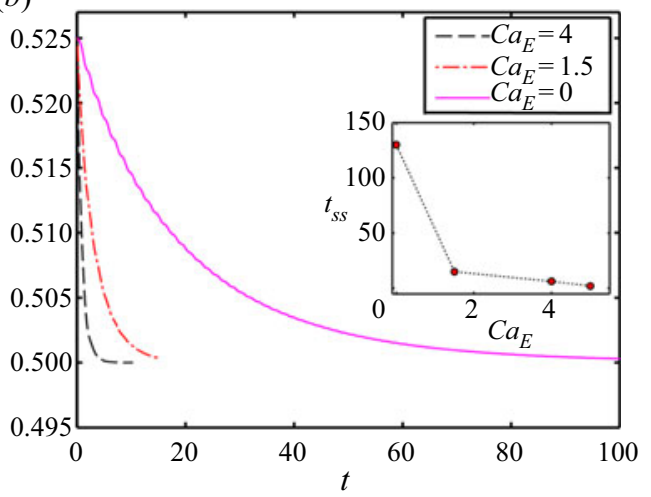

FIgURE 3. (a) Cross-stream migration characteristic of the droplet in oscillatory microflow, (b) Temporal variation of the transverse position of the droplet's centroid. The variation of $t_{s s}$ with $C a_{E}$ is shown in the inset of figure 3(b). Important simulation parameters are $(S, R)=$ (2, 0.5), $C a=0.3, Y_{d}=0.525, \rho_{r}=1, \lambda=1, a=0.3, R e=0.1$ and $S t=2$.

sorting process takes place very sluggishly, bearing adverse consequences on the net throughput. The findings of figure 3 illustrate that the presence of the axial electric field offers a remedy to overcome this constraint to a large and controllable extent.

Similarly, figure 4 illustrates the electric-field-induced modification of the cross-stream migration of the droplet for another physical system, henceforth termed as system B. The distinctive hallmark of system B, as compared with system A, is as follows: the conductivity ratio $(R)$ of the system is higher than the permittivity ratio $(S)$ that essentially creates the electrohydrodynamic flow from poles to equators. Relevant dimensionless properties are $(S, R)=(0.5,2), \rho_{r}=1$ and $\lambda=1$. From figure $4(a)$, it is evident that the axial oscillations of the droplet before reaching the centreline are amplified for moderate values of $C a_{E}$ (=1 and 1.5). Hence, the value of $t_{s s}$ increases as depicted in figure $4(b)$. If we slightly increase the value of $C a_{E}(=1.52)$, the droplet achieves its steady-state transverse position almost immediately $\left(t_{s s}=0.583\right)$. In this case, the ultimate position of the droplet $(=0.5252)$ is nearly equal to its initial position $(=0.525)$. For this value of $\mathrm{Ca}_{E}$, the axial oscillation of the droplet before reaching steady-state transverse position disappears completely. However, after reaching the steady-state transverse position, the droplet undergoes axial oscillations along a horizontally straight pathway. If we further increase the value of $C a_{E}(=2)$, the steady-state transverse position of the droplet shifts towards the upper wall and the axial oscillations of the droplet before reaching the final position again amplifies. Accordingly, the magnitude of $t_{s s}$ increases. However, if we raise the value of $C a_{E}$ beyond a threshold limit $\left(C a_{E}=5\right)$, not only do the axial oscillations attenuate, but the droplet also moves towards the nearest wall at a faster rate. Hence the magnitude of $t_{s s}$, again, reduces. Therefore, for this leaky dielectric system, the magnitude of $t_{s s}$ varies non-monotonically with the values of $\mathrm{Ca}_{E}$. This phenomenon has immense importance in efficient and rapid manipulation of biological and non-biological deformable entities at will in a microfluidic system, constrained by its axial length.

We now discuss the physical reasoning behind these observations. In a steady pressure-driven flow, the droplet's cross-stream motion in microchannel occurs owing to (i) hydrodynamic force $\left(F_{H}\right)$, originating out of the streamline curvature and deformed shape of the droplet, and (ii) non-inertial lift force $\left(F_{L}\right)$, stemming from the wall effects. However, when the droplet is subjected to oscillatory pressure-driven flow, $F_{H}$ becomes 
(a)

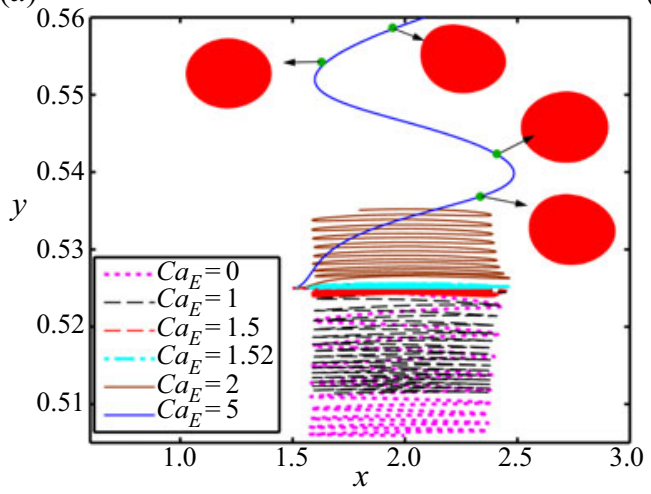

(b)

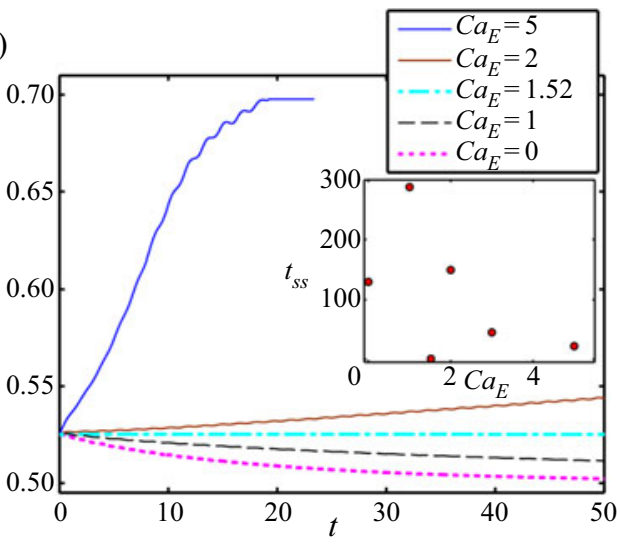

FIGURE 4. (a) Cross-stream migration characteristic of the droplet in an oscillatory microflow, (b) Temporal variation of the transverse position of the droplet's centroid. The variation of $t_{s s}$ with $C a_{E}$ is shown in the inset of figure $4(b)$. Important simulation parameters are $(S, R)=$ $(0.5,2), C a=0.3, Y_{d}=0.525, \rho_{r}=1, \lambda=1, a=0.3, R e=0.1$ and $S t=2$.

oscillating in nature and creates oscillations in the droplet that hinders the droplet's motion in the transverse direction. The imposed oscillation is quantitatively characterized by the dimensionless oscillation frequency or the Strouhal number, St. For high values of $S t$, the imposed oscillation arrests the transverse motion of the droplet to a considerable extent. However, in the presence of an electric field, two additional forces also act on the droplet and modify droplet's migration characteristics in a dramatic fashion. These forces are (i) dielectrophoretic force $\left(F_{D}=-1 / 2 \cdot E^{2} \cdot \nabla \varepsilon\right)$ and (ii) electrohydrodynamic force $\left(F_{E H D}\right)$.

Although variations in electrical permittivity trigger dielectrophoretic forces, the asymmetric distribution of electric shear-driven flow circulation generates electrohydrodynamic forces. The net dielectrophoretic force always attempts to drive the droplet towards the wall in closer vicinity, irrespective of the electrical properties of the system. This phenomenon can be justified through figure 5(a), where the variation of $E^{2}$ is plotted along a vertical line, drawn from the bottom wall to top wall and going through the centroid of the droplet.

Figure 5(a) illustrates that, for system A, the strength of $E^{2}$ is higher at the bottom side of the droplet as compared with its top side. Again, the magnitude of the $E^{2}$ is lower in the ambient fluid with respect to the inside fluid of the droplet. Similarly, for system $\mathrm{B}$, the strength of $E^{2}$ is higher and lower at the bottom half and the top half of the droplet, respectively, and the strength of $E^{2}$ is higher outside the droplet with respect to its inner region. This symmetry breaking in $E^{2}$ creates an imbalance in dielectrophoretic force at the two surfaces, producing a translational motion of the droplet in the upward direction (Esmaeeli 2016). However, the net $F_{E H D}$ can drive the droplet toward the wall or channel centreline based on the direction of electric-shear-driven flow circulation around the droplet which is further determined from the conductivity ratio $(R)$ and permittivity ratio $(S)$. Under a steady axial electric field, for system A, the direction of the flow circulation is from the equator to the poles as depicted in figure $5(b)$. Thus, for system $\mathrm{A}$, the net $F_{E H D}$ tries to drive the droplet toward the centreline. Briefly, in system A, when a droplet is placed above the centreline, the electric field creates two asymmetric vortex pairs inside the droplet as depicted in figure $5(b)$. Therefore, the $F_{E H D}$, arising because of the inner vortex at the top half and bottom half (with respect to the horizontal line of 

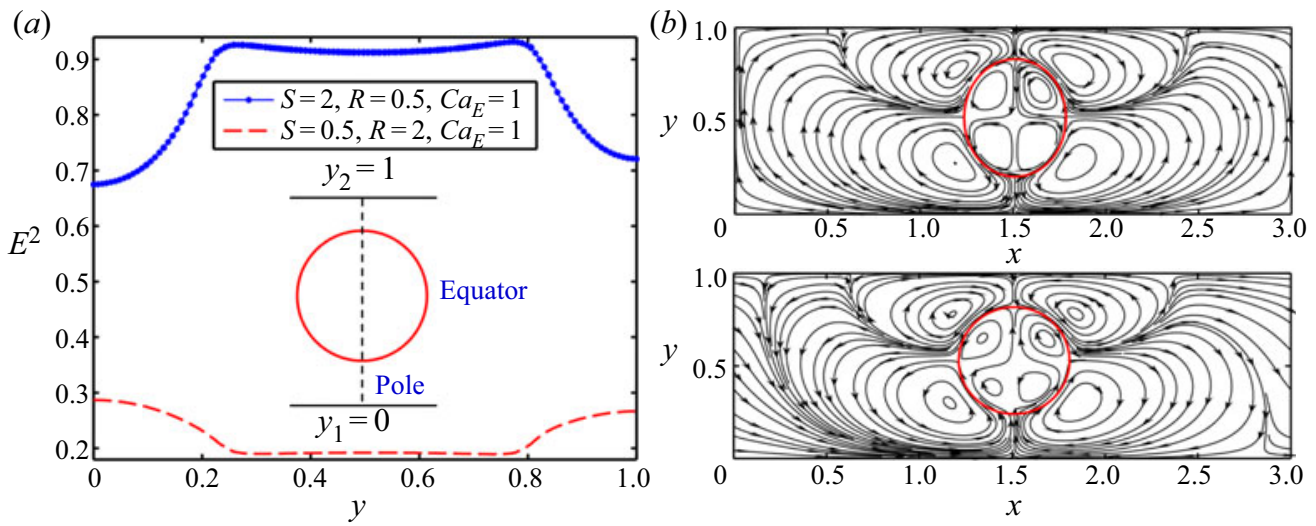

(c)

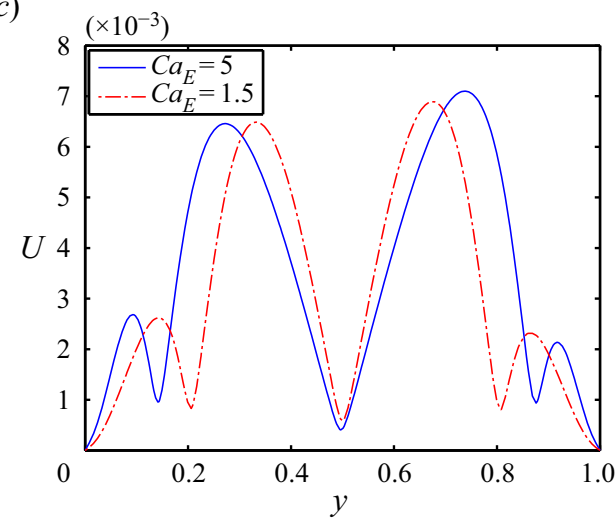

FIGURE 5. The influence of electric field on the droplet. (a) Variation of $E^{2}$ along a vertical straight line passing from lower wall to the upper wall through the centroid of the droplet. Streamline pattern of flow circulation, formed in the presence of an electric field for $(b)$ system A having $(S, R)=(2,0.5)$ and $(c)$ system B having $(S, R)=(0.5,2)$. For $(a-c)$, the value of $C a_{E}$ is 1.5. (d) Variation of the magnitude of velocity along a probe passing through the centroid of the droplet and drawn from the lower wall to the upper wall for system A having $(R, S)=$ $(0.5,2)$. Other parameters are $Y_{d}=0.525, \rho_{r}=1, \lambda=1, a=0.3, t=2.5$ and $R e=0.1$.

symmetry) of the droplet, attempts to drive the droplet toward the centreline and nearby wall, respectively. Figure $5(d)$ shows that the magnitude of the velocity is greater inside the droplet in the upper half. Hence, the strength of the generated $F_{E H D}$ owing to inner vortex pairs at the upper half is significantly higher with respect to that in the lower half.

Owing to this fact, the net $F_{E H D}$ due to the dynamics of the inner vortex attempts to set the droplet in motion towards the channel centreline. Similar to the inner vortex pair, counter-rotating pairs of outer vortices are generated just outside the droplet. The $F_{E H D}$ owing to outer vortex pairs at the top side or bottom side attempts to pull the droplet towards the nearest wall and the channel centreline, respectively. From figure $5(d)$, we observe that the strength of the outer vortex pair at the bottom half of the droplet is greater than the top half. Hence, the net $F_{E H D}$ caused by the dynamics of the outer vortex also pulls the droplet towards the channel centreline. One can infer that the resultant effect of the net $F_{E H D}$ due to the dynamics of vortices drives the droplet towards the centreline. It is worth mentioning that the strength of the net $F_{E H D}$ depends on the strength of the flow 

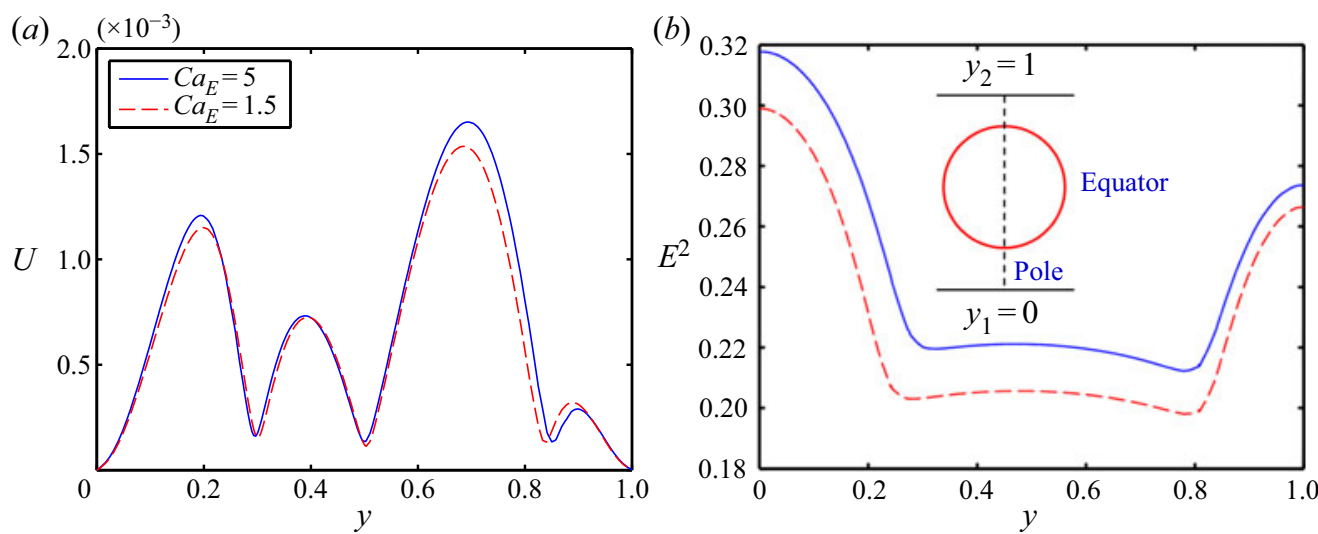

FIGURE 6. (a) Variation of the magnitude of velocity along a probe passing through the centroid of the droplet and drawn from the lower wall to the upper wall for system B. (b) Variation of $E^{2}$ along a vertical straight line passing from the lower wall to the upper wall through the centroid of the droplet for system B. Other parameters are $Y_{d}=0.525,(R, S)=(2,0.5), a=0.3, t=2.5$, $\operatorname{Re}=0.1$ and $\lambda=1$.

circulation, which again relies on the strength of the electric field. Figure $5(d)$ shows that for system A, with the enhancement of $C a_{E}$, the strength of the flow circulations in the inner vortex at the top half and outer vortex at the bottom half increases, which amplifies the strength of the net $F_{E H D}$. Thus, for system A, the direction and the patterns of the droplet motion are determined by the interplay among $F_{H}, F_{L}, F_{D}$ and $F_{E H D}$. For system A, at higher values of $C a_{E}$, the strength of the net $F_{E H D}$ increases drastically and becomes dominant over the other forces that drive the droplet to the centreline at a faster rate in an unwinding pathway.

Similar to system A, in system B the interplay among the $F_{H}, F_{L}$, net $F_{D}$ and net $F_{E H D}$ also dictates the direction and pattern of the cross-stream motion of the droplet. However, for system B, the direction of electrohydrodynamic flow takes place from poles to the equators as depicted in figure 5(c). Therefore, the net $F_{E H D}$ tries to drive the droplet towards the nearby wall. In short, in system B, when a droplet is kept above the centreline, the electric field creates two asymmetric vortex pairs inside the droplet, as illustrated in figure 5(c). Hence, $F_{E H D}$, arising because of the inner vortex at the top half and the bottom half (with respect to the horizontal line of symmetry) of the droplet, attempts to move the droplet toward the adjacent wall and centreline, respectively. Along with inner vortex pairs, two pairs of counter-rotating outer vortices are also formed. The $F_{E H D}$ originating out of the outer vortex pairs at the upper half of the droplet tries to push the droplet towards the centreline, whereas the $F_{E H D}$ owing to the outer vortex pair at the bottom half of the droplet tries to push the droplet towards the nearby wall.

Figure 6(a) shows that the strength of inner vortex pairs and the outer vortex pairs is high at the upper half and lower half of the droplet, respectively. Therefore, the net $F_{E H D}$ owing to the dynamics of the vortex pairs attempts to move the droplet towards the nearby wall. Furthermore, as we mentioned earlier, the net $F_{D}$ arising owing to the asymmetric distribution of $E^{2}$ also attempts to push the droplet towards the nearby wall. On the other hand, the forces $F_{L}$ and $F_{H}$ attempt to move the droplet towards centreline, opposing these effects. At a moderate value of $C a_{E}(=1.5)$, figure 6(a) shows that the strength of the flow circulation is comparatively weak. Therefore, the strength of net $F_{E H D}$ is also relatively low. 


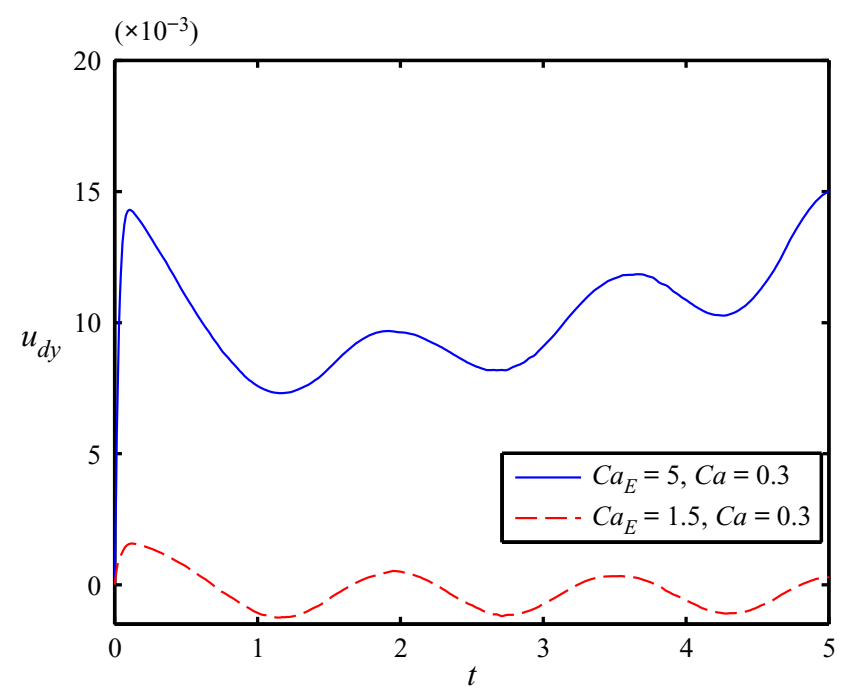

FIGURE 7. Effect of the electric capillary number on $u_{d y}$ (y-component of the velocity of the droplet) in the combined presence of a steady axial electric field and background oscillatory flow. Others parameter are $(S, R)=(0.5,2), Y_{d}=0.525, a=0.3, \lambda=1$ and $R e=0.1$.

Similarly, figure 6(b) also confirms that the magnitude of $E^{2}$ is also comparatively lower for $C a_{E}=1.5$. Hence, for this value of $C a_{E}$, the united strength of net $F_{E H D}$ and $F_{D}$ is also not significantly high. Therefore, in this scenario, the combined effect of $F_{H}$ and $F_{L}$ remains dominating in nature. Owing to the opposing nature of net $F_{E H D}$ and $F_{D}$, they attempt to lessen the combined effect of $F_{H}$ and $F_{L}$. As a result, for $C a_{E}=1.5$, the transverse component droplet's velocity $\left(u_{d y}\right)$ towards the centreline reduces, as depicted in figure 7. Because the magnitude of $u_{d y}$ is low for $C a_{E}=1.5$ and the considered value of the $S t$ is considerably high $(S t=2)$, the droplet takes greater time to reach the centreline and exhibits more number of oscillations before arriving at the centreline. Therefore, the magnitude of $t_{s s}$ also increases. If we further increase the value of $C a_{E}$, the magnitude of net $F_{E H D}$ and $F_{D}$ also increases. At $C a_{E}=1.52$, the united effect of net $F_{E H D}$ and $F_{D}$ nullifies the combined strength of $F_{H}$ and $F_{L}$ almost instantaneously, and the droplet immediately attains a steady-state position. Therefore, the magnitude of $t_{s s}$ also reduces drastically. For further increase in the value of $C a_{E}(=2)$, the integrated strength of net $F_{E H D}$ and $F_{D}$ becomes dominant in nature and shifts the steady-state transverse position towards the upper wall. In this scenario, the axial oscillations of the droplet before reaching the steady-state position again amplify and the magnitude of $t_{s s}$ also increases. However, on further increase of $C a_{E}(=5)$, the magnitude of strength of flow circulation and the magnitude of $E^{2}$ enhance significantly, as shown in figures $6(a)$ and $6(b)$, respectively. Therefore, the integrated strength of net $F_{E H D}$ and $F_{D}$ enhances markedly. This not only arrests axial oscillations in the cross-stream motion, but also increases the magnitude of $u_{d y}$ drastically (as shown in figure 7), which leads to the faster rate of droplet motion toward the wall closest to it.

A critical assessment of the intricate interplay of electromechanical and hydrodynamic forces described previously reveals that the combined effect of the steady axial electric field and the oscillatory pressure-gradient-driven flow on the droplet's drift velocity is not necessarily a mere linear superposition of the droplet's velocity obtained in an oscillatory 


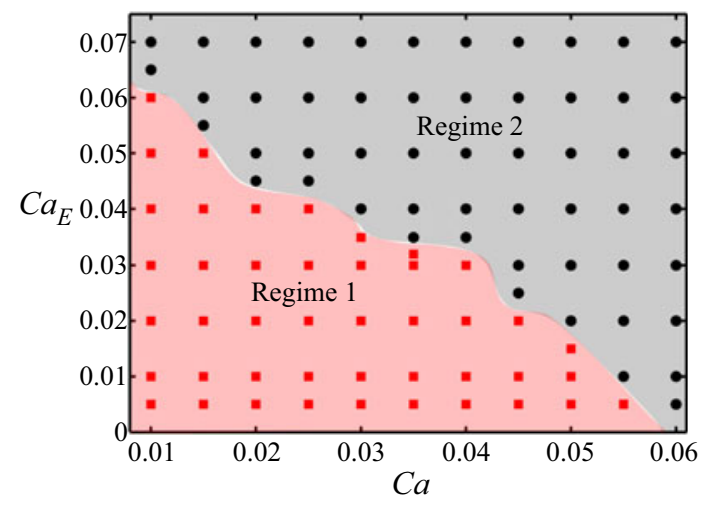

FIGURE 8. Regime plot showing two distinct regimes based on the values of $\mathrm{Ca}$ and $\mathrm{Ca}$. Regime 1: the effects of oscillatory flow and axial electric field may be combined by linear superposition of the respective drift velocities. Regime 2: the linear superposition fails. Others parameter are $(S, R)=(0.5,2), Y_{d}=0.525, a=0.3, \lambda=1$ and $R e=0.1$.

flow and steady axial electric field, separately. This is due to the two-way nonlinear coupling between electric potential and flow field mediated by unique shape modulation: the applied electric field modifies the fluid flow by developing Maxwell stress at the interface of the droplet; dynamical evolution of the droplet's shape being unknown $a$ priori. Again, this flow field causes the deformation of the droplet, which, in turn, modifies the electric potential distribution. Owing to the fact that the intrinsic cause of the coupled nature of the electromechanics and hydrodynamics is the shape deformation of the droplet, the resultant effect of oscillatory flow and electric field can be combined by simply adding together the respective drift velocities only in small deformation limits. Although this represents the essential qualitative physics, a more quantitative depiction is presented in the following.

In an effort to offer a quantitative perspective of the conceptual foundation delineated previously, we next depict a regime plot across the $C a-C a_{E}$ parameter space, in figure 8, to probe the extent of validity of linear superposition of the drift velocities obtained by considering the individual forcing parameters separately. The linear superposition approach is considered to validate the outcome from the combined effect when the difference between them is less than $0.5 \%$. The regime plot clearly shows two distinct regimes: regime 1, comprising 'rectangular data points' where the linear superposition is valid; and regime 2, comprising 'circular data points', where it fails. Evidently, for low values of $C a$ (typically, less than 0.01 ), the linear superposition works up to a comparatively high value of $C a_{E}(=0.06)$. This may be attributed to the fact that for such low values of $\mathrm{Ca}$, the viscous stress-induced droplet deformation is much less. As the magnitude of $C a$ increases, this upper limit of $C a_{E}$ (for the validity of the linear superposition) progressively reduces, stemming from a nonlinear interplay between the electromechanics and hydrodynamics as mediated by the droplet deformation. Beyond $C a=0.06$, the viscous stress-induced deformation of the droplet becomes so large that the linear superposition deems completely invalid for any finite, non-zero values of $\mathrm{Ca}_{E}$.

Next, we construct a regime diagram across the $C a_{E}-S t$ parameter space for system $\mathrm{A}$, as shown in figure 9, in an effort to unveil of coupling between the imposed flow oscillation and electrical forcing. In the figure, region A containing blue-coloured circular markers denotes oscillatory downward motion of the droplet, whereas the red-coloured 


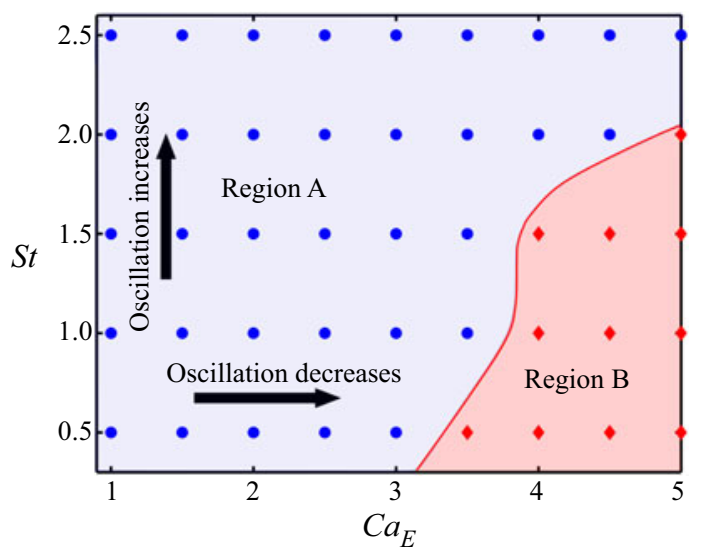

- Oscillatory downward

Non-oscillatory downward

FIgURE 9. Regime plot based on the values of $\left(C a_{E}, S t\right)$. Other parameters are $(S, R)=(2,0.5)$ $C a=0.3, a=0.3, \operatorname{Re}=0.1, \lambda=1$ and $Y_{d}=0.525$.

diamond-shaped markers in region B represent oscillation-free downward motion of the same.

Figure 9 further suggests that, at low values of the $S t$, the transition of the droplet motion from oscillatory to non-oscillatory state takes place at comparatively low values of $C a_{E}$. However, on increasing the value of $S t$, the magnitude of $C a_{E}$ necessary for converting the droplet's motion from oscillatory to oscillation-free nature increases. For substantially high values of $S t$, the droplet undergoes oscillatory downward motion for any possible values of $C a_{E}$. Competing influence of the electric-field-induced net $F_{E H D}$ and the imposed oscillation induced net $F_{H}$ is responsible for such phenomena.

\subsubsection{Effect of domain confinement on the cross-stream motion of the droplet}

Figure 10 shows the effect of domain confinement on the cross-stream motion of the droplet under the combined influence of steady axial electric field and the background oscillatory flow. The degree of confinement is denoted by the domain confinement ratio $\left(W c=2 a^{*} / H^{*}\right)$. In the present study, we have altered the magnitude of $W c$ by varying the radius of the droplet keeping other parameters intact. Hence, the effect of the size of the droplet is taken care of by the domain confinement ratio. One primary contribution of the domain confinement manifests via alterations in the hydrodynamic interactions. This alters the balance of the pertinent forces via electrohydrodynamic coupling, triggering transients and cross-stream migration of droplets over small scales, unlike the unbounded scenario (where $a \ll H$ or $W c \ll 1)$ ).

In addition, figure $10(a)$ shows that the droplet undergoes oscillatory cross-stream motion following a zig-zag pathway in a weakly confined domain. However, in a tightly confined domain, the axial oscillations before reaching the centreline attenuate. Beyond a threshold value of $W c$, the droplet moves to the channel centreline in an uncurling pathway without making axial oscillations. Again, figure $10(b)$ illustrates that the magnitude of $t_{s s}$ also reduces with enhancement of the domain confinement ratio, which dictates more rapid settling of the droplet to its final steady state in highly confined domains.

We now explain the physics behind the observed phenomena described previously. For the considered values of electrical properties, the electrohydrodynamic flow circulation is directed from the equator to poles, as shown in figures $11(a)$ and 11(b). Hence, the 
(a)

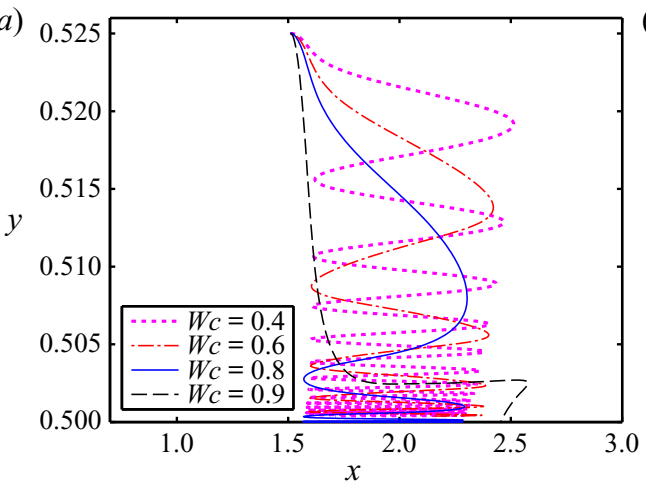

(b)

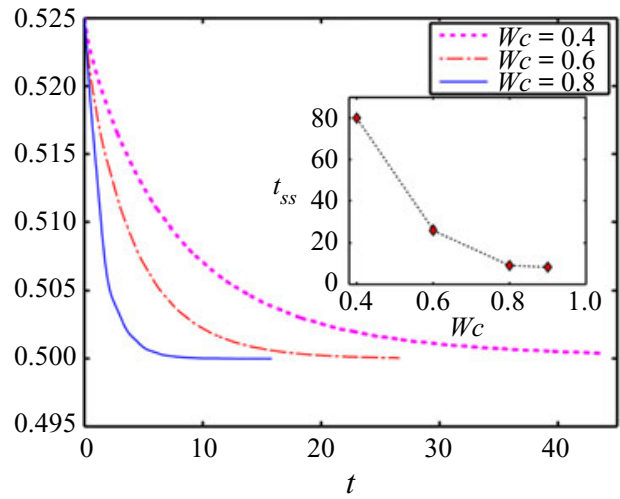

FIGURE 10. Effect of domain confinement on $(a)$ the migration characteristic of the droplet and (b) the temporal variation of the transverse position of the droplet's centroid. The variation of $t_{s S}$ with $W c$ is shown in the inset of $(b)$. Other parameters are $(S, R)=(2,0.5), C a=0.3, C a_{E}=1.5$, $a=0.3, \operatorname{Re}=0.1, \lambda=1, Y_{d}=0.525$ and $S t=2$.

electrohydrodynamic force owing to inner vortex pair at the upper half and outer vortex pairs at the lower half of the droplet attempts to shift the droplet towards the channel centreline. On the other hand, the electrohydrodynamic force owing to the inner vortex pair at the lower half and outer vortex pair at the upper half attempts to push the droplet towards the domain wall. Figure 11(c) depicts that the magnitude of the velocity of the inner vortex is higher at the upper half, as compared with the the lower half, and the difference in the magnitudes of velocity enhances with the increase in relative domain confinement. The consequent increase in the net $F_{E H D}$ attempts to drive the droplet towards the centreline. In a similar way, the net $F_{E H D}$ attributable to the outer vortex also increases with the enhancement of relative domain confinement. Furthermore, with an increase in the relative domain confinement, the strength of $F_{L}$ also enhances. This effect is compounded by enhanced $F_{H}$ in more tightly confined domains. An elevated combined strength of net $F_{E H D}, F_{L}$ and $F_{H}$, thus, becomes capable of moving the droplet rapidly in an uncurling pathway, with enhancements in the relative domain confinement.

\subsubsection{Effect of electrical properties of the system on the cross-stream motion of the droplet}

The alteration of the cross-stream motion of the droplet for different values of $(R / S)$ is plotted in figure 12. Figure 12(a) reveals that, with increments in $R / S$ ratio, the axial oscillation of the droplet enhances before reaching the centreline and the transverse extent traversed by the droplet is small. On decreasing the value of $R / S$, we observe a reduction in the axial oscillations of the droplet, and the transverse distance travelled by the droplet also increases. For comparatively lower values of $R / S$, the droplet moves to the centreline without undergoing axial oscillations. Figure $12(b)$ further corroborates that the magnitude of $t_{s s}$ decays with the lowering of $R / S$.

The exclusive dependence of the transverse migration characteristics of the droplet on the relative electrical properties can be explained as follows. In the confined domain, the forces $F_{L}$ and $F_{H}$ attempt to move the droplet towards the domain centreline. On the other hand, for $R / S>1$, the direction of electrohydrodynamic flow takes place from the poles to the equator, and the net $F_{E H D}$ owing to the inner vortex pairs at the upper half and outer vortex pair at the lower half of the droplet tries to drive the droplet 

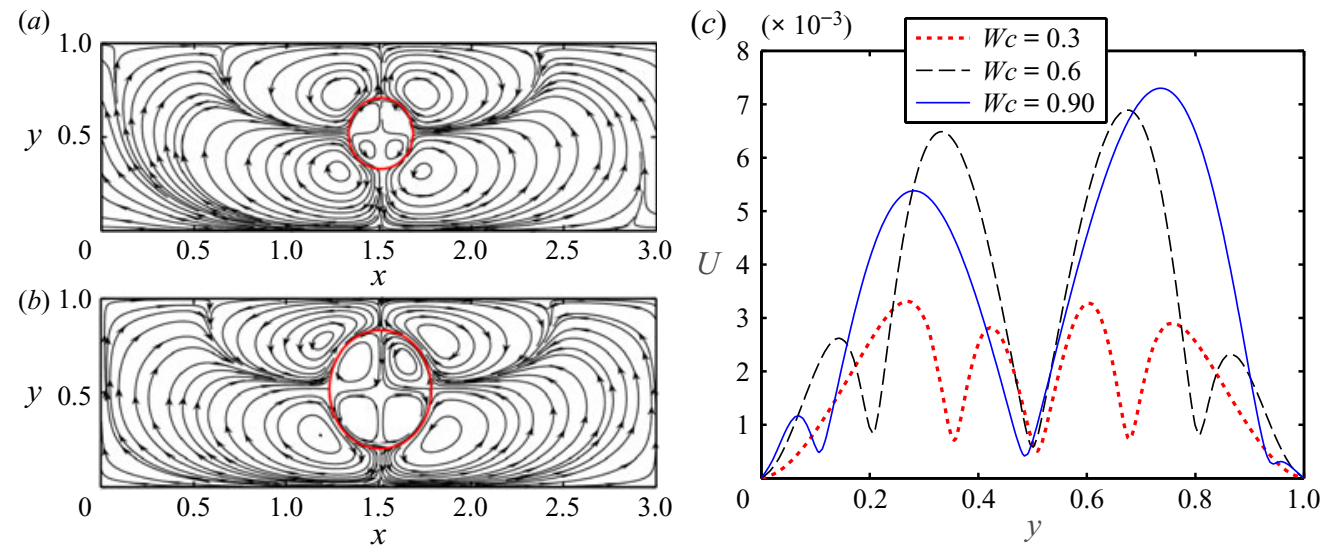

FIGURE 11. Streamline pattern of flow circulation, formed in the presence an electric field for (a) $W c=0.3$ and (b) $W c=0.6$. (c) Distribution of the magnitude of velocity along a straight line passing through the centreline and drawn from the lower wall to the upper wall. Other parameters are $(S, R)=(2,0.5), C a=0.3, C a_{E}=1.5, a=0.3, R e=0.1, \lambda=1, t=2.5, Y_{d}=0.525$ and $S t=2$.

(a)

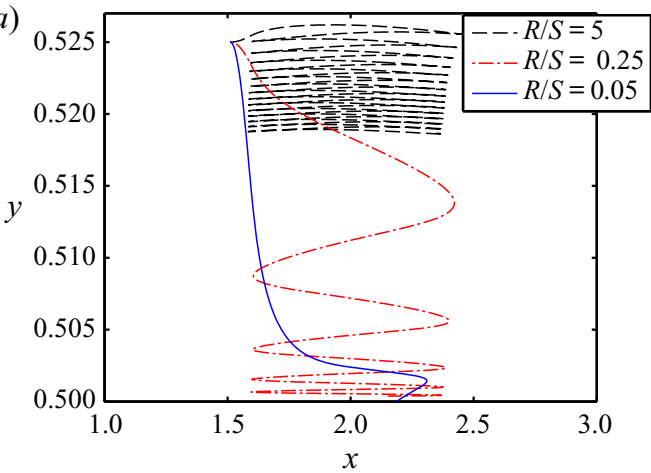

(b)

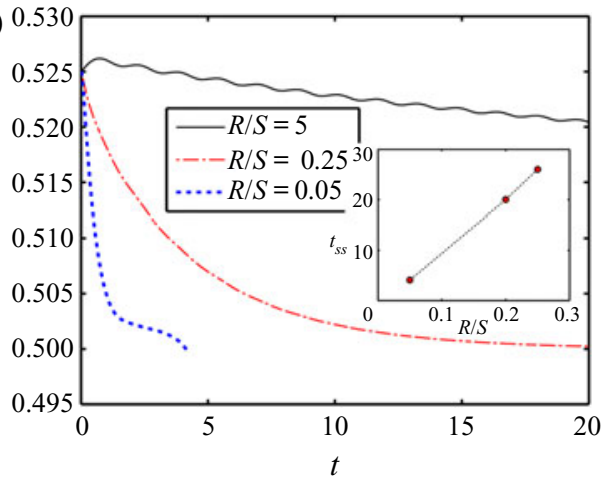

FIGURE 12. Effect of the electrical property ratio on $(a)$ the cross-stream migration of the droplet and (b) the temporal variation of the transverse position of the droplet's centroid. The variation of $t_{s s}$ with $R / S$ is shown in the inset of $(b)$. Other parameters are $R=0.5, C a=0.3$, $C a_{E}=1.5, a=0.3, R e=0.1, \lambda=1, Y_{d}=0.525$ and $S t=2$.

towards the wall nearest to it. Similarly, the net $F_{D}$ also tries to shift the droplet in a similar direction. The combined consequences of $F_{D}$ and $F_{E H D}$ neutralize the combinatorial effect of $F_{L}$ and $F_{H}$ to a large extent, which leads to amplified axial oscillations of the droplet and decrease in the transverse extent traversed by the same. However, for $R / S<1$, the direction of electrohydrodynamic flow takes place from equators to poles and its strength is significantly large for $R / S \ll 1$. Therefore, the net $F_{E H D}$ tries to move the droplet towards the channel centreline and its strength is also considerable. The combined effect of net $F_{E H D}, F_{L}$ and $F_{H}$ drives the droplet to the centreline in an uncurling pathway, without incurring axial oscillations.

Figure 13 shows a regime diagram mapping three distinct regimes of droplet migration, depending on the values of $(R, S)$ : region I, comprising 'rectangular data points', where the droplet undergoes oscillatory upwards motion towards the wall near to it; region II, 


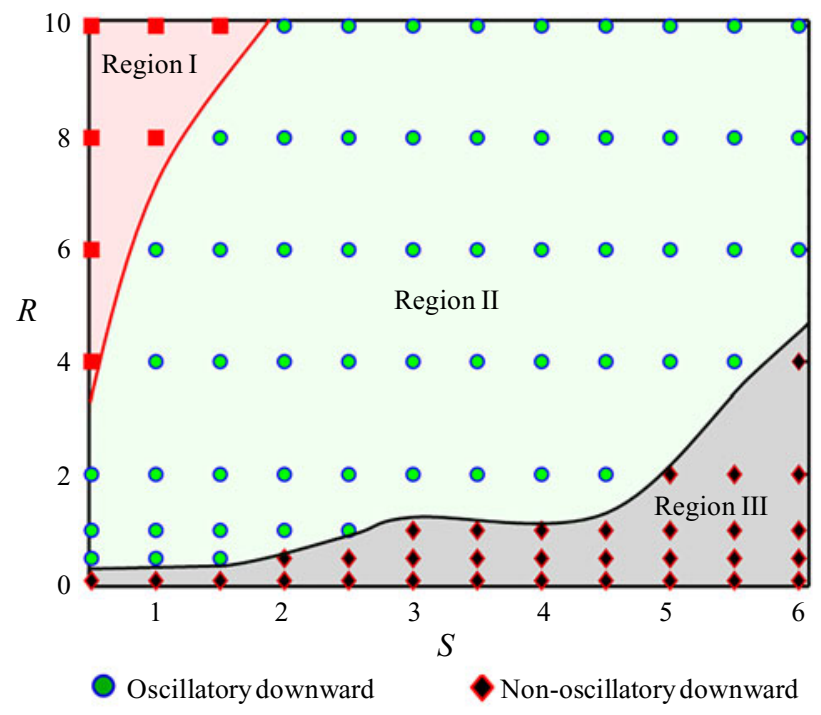

Oscillatory upward

FIGURE 13. Regime plot based on the values of $(S, R)$. Other parameters are $C a=0.3$, $C a_{E}=2, a=0.3, R e=0.1, Y_{d}=0.525, \lambda=1, W c=0.6$ and $S t=2$.

containing 'circular data points', representing the oscillatory downward motion of the droplet towards channel centreline; region III, comprising 'diamond-shaped data points', where the droplet moves to the centreline without following axial oscillations. In region I, the combined strength of net $F_{D}$ and $F_{E H D}$ overweighs the combined effect of $F_{H}$ and $F_{L}$ because $R \gg S$, and it leads to the motion of the droplet towards the nearby wall. On decreasing the value of $R$ and increasing the value of $S$, the dynamics of droplet motion shifts from regime I to regime II. In regime II, for the data points satisfying $R / S>1$, the integrated effect of net $F_{D}$ and $F_{E H D}$ is not strong enough to outweigh the combined effect of $F_{H}$ and $F_{L}$. Thus, the droplet moves towards the centreline following a zig-zag pathway.

On the other hand, in region II, for the data points satisfying $R / S<1$, the net electrohydrodynamic force acts in the direction of $F_{H}$ and $F_{L}$ and their integrated strength drives the droplet towards the centreline in a zig-zag pathway. For further decrease in the value of $R$, there is a cross-over from regime II to regime III. In region III, because $R / S \ll 1$, the strength of net $F_{E H D}$ is very high. This $F_{E H D}$, along with $F_{H}$ and $F_{L}$, attenuates the axial oscillation in the cross-stream motion of the droplet before reaching the channel centreline.

\subsection{Experimental investigation}

\subsubsection{Fabrication of microfluidic device}

For experimental verification of the essential theoretical findings of this work, we have first fabricated a master-mould by a conventional photolithography technique and then performed a standard soft-lithography process to obtain the polydimethylsiloxane (PDMS) device from the mould (Dey et al. 2015; Santra et al. 2018a). After the soft-lithography process, the solidified PDMS pattern is separated from the mould on sufficient curing, and arrangements of the inlet and outlet port are made. The closing side of the microfluidic device is patterned with gold electrodes using a shadow mask and DC sputtering coater 


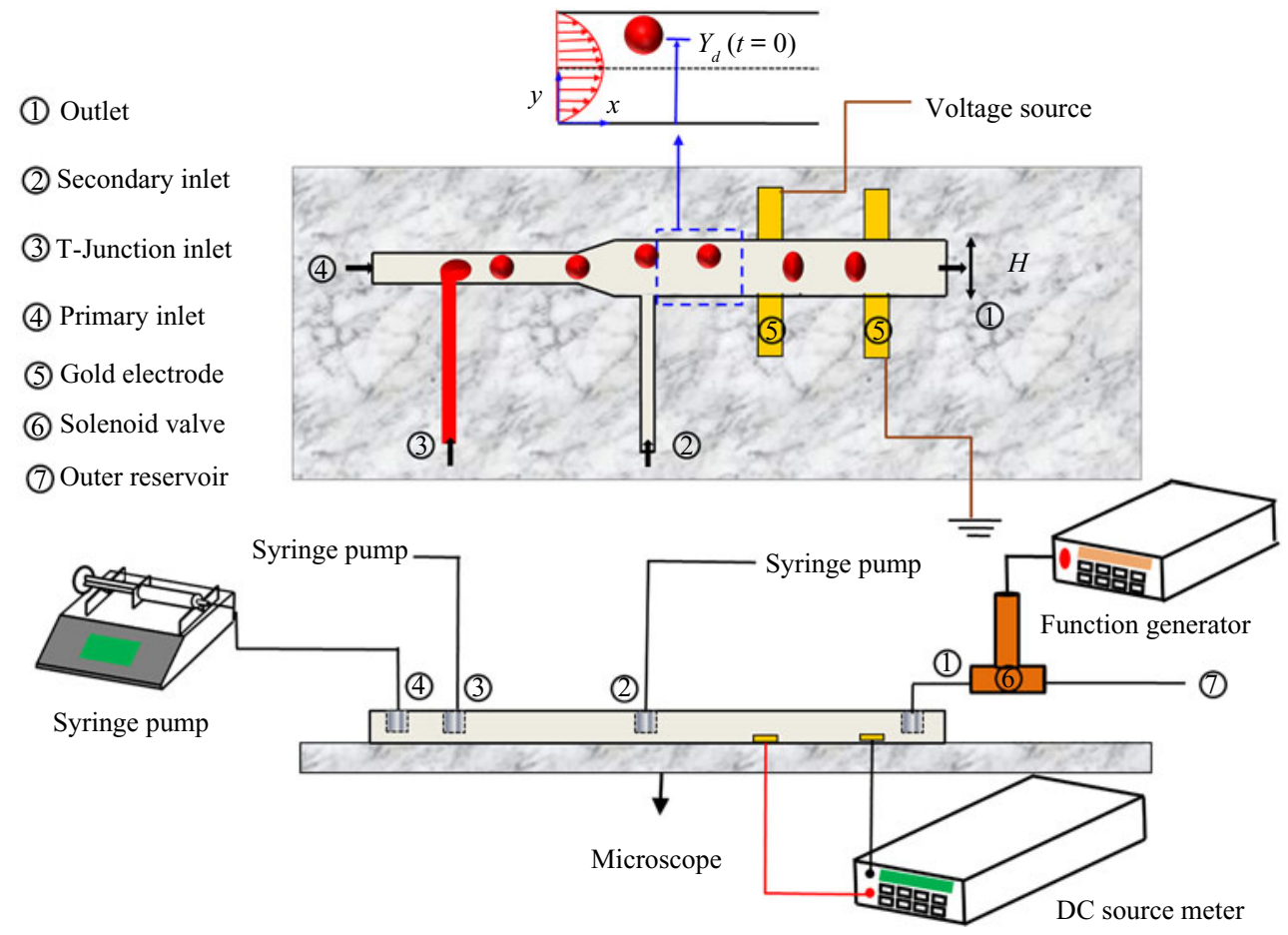

FIGURE 14. Schematic illustration of the experimental set-up.

(Ted pella, Cressington 108, USA). The patterned glass substrates have then been bonded to the PDMS microchannel by oxygen-plasma bonding (Dey et al. 2015).

\subsubsection{Experimental set-up and methodology}

Figure 14 shows the schematic illustration of the experimental set-up. The experimental set-up comprises a PDMS-based T-shaped microchannel and gold electrodes assembly. The T-shaped microchannel has been used to produce monodispersed droplets at the desired frequency. After the production of the droplets at the T-junction, they are carried out by the continuous phase in the outlet direction and at the diverging section. The positions of droplets are shifted to an off-centre position owing to hydrodynamic lift force produced by the flow from the secondary inlet. At this diverging section, a uniform DC electric field is applied in the direction of flow. For supplying the DC voltage, we have placed two gold electrodes in the axial direction, connected with a DC power supply (Keithley- 2410). The electrodes are placed on one wall of the device (bottom glass substrates) and the distance between the electrodes is $5 \mathrm{~mm}$.

In the experimental analysis, we have estimated the unperturbed electric field strength from the following equation:

$$
E_{\infty}^{*}=-\frac{\phi_{1}^{*}-\phi_{2}^{*}}{L^{*}}
$$

Here, $\phi_{2}^{*}$ and $\phi_{1}^{*}$ are the electric potential at the high-voltage electrode and grounded electrode $\left(\phi_{1}^{*}=0\right.$ in the analysis), respectively, and $L^{*}$ is the distance between the electrodes. It is worth mentioning that, in the presence of a droplet, the electric field in 
the droplet region is non-uniform owing to the disparity in the electrical properties of the droplet and the suspending fluid.

For a representative case study, the microchannel depth is $410 \mu \mathrm{m}$, whereas its width $(H)$ turns out to be 400 and $480 \mu \mathrm{m}$, for systems A and B, respectively (measured by a Dektak 150 surface profiler). For our specific experiments reported here, these systems are described as follows: (a) system A having $S>R$, where silicone oil (with $\epsilon_{i}=3.43 \times$ $10^{-11} \mathrm{~F} \mathrm{~m}^{-1}, \sigma_{i}=9.26 \times 10^{-11} \mathrm{~S} \mathrm{~m}^{-1}, \mu_{i}=2.046 \times 10^{-2} \mathrm{~Pa} \cdot \mathrm{s}$ and $\left.\rho_{i}=1023 \mathrm{~kg} \mathrm{~m}^{-3}\right)$ and sunflower oil (with $\epsilon_{e}=2.88 \times 10^{-11} \mathrm{~F} \mathrm{~m}^{-1}, \sigma_{e}=4.74 \times 10^{-9} \mathrm{~S} \mathrm{~m}^{-1}, \mu_{e}=4.9 \times 10^{-2} \mathrm{~Pa} \cdot \mathrm{s}$ and $\rho_{e}=921 \mathrm{~kg} \mathrm{~m}^{-3}$ ) are used as the dispersed phase and continuous phase, respectively; (b) system B having $R>S$, where deionized (DI) water (with $\epsilon_{i}=6.90 \times 10^{-10} \mathrm{~F} \mathrm{~m}^{-1}$, $\sigma_{i}=5.49 \times 10^{-6} \mathrm{~S} \mathrm{~m}^{-1}, \mu_{i}=1 \times 10^{-3} \mathrm{~Pa} \cdot \mathrm{s}$ and $\left.\rho_{i}=998 \mathrm{~kg} \mathrm{~m}^{-3}\right)$ and silicone oil are employed as the dispersed phase and continuous phase, respectively. The interfacial tension between the DI water and silicone oil is $33 \mathrm{mN} \mathrm{m}^{-1}$ (Peters \& Arabali 2013), whereas its magnitude for silicon oil and sunflower oil is $1.8 \mathrm{mN} \mathrm{m}^{-1}$ [measured using a pendant drop method integrated with a goniometer (250 G1, Ramé-hart, Germany)].

To supply the continuous and dispersed fluids in the microchannel, three syringe pumps (Harvard PHD 2000) are used. The flow rates at the T junction inlet $\left(Q_{d}\right)$, primary inlet $\left(Q_{1 c}\right)$ and secondary inlets $\left(Q_{2 c}\right)$ are varied within a range of $20-50 \mu 1 \mathrm{~h}^{-1}, 130-150$ $\mu 1 \mathrm{~h}^{-1}$ and 30-60 $\mu 1 \mathrm{~h}^{-1}$, respectively. To achieve a time-varying flow field, a solenoid pinch-off valve (Cole-Parmer) is coupled with the outflow flexible Tygon $₫$ tubing. The solenoid valve is actuated by employing $8 \mathrm{~V}_{p p}$ (peak-to-peak voltage) sinusoidal waveform with $2 \mathrm{~Hz}$ frequency generated from a function generator (Agilent 33220A).

\subsubsection{Cross-stream migration characteristic of the droplet}

Here, we compare the experimental results with the corresponding theoretical predictions. For comparing these results, we have directly input the specific pressure profile as obtained from the experimental analysis into the numerical simulations.

For measuring the differential pressure across the channel length, a differential pressure sensor (Honeywell FDW) coupled with a data acquisition system [DAQ (NI 6009, National Instruments)] is used as shown in figure 15(a). For using the differential pressure sensor, we have selected two pressure tapping points $1 \mathrm{~cm}$ apart along the microchannel length. The DAQ system, having an interface with Lab PC running Labview (National Instruments) program, takes the raw voltage from the pressure sensors and converts it into the relevant physical unit depending on the instrument calibration chart mentioned in the program. For instance, figures $15(b)$ and $15(c)$ show the applied voltage waveform and obtained differential pressure waveform, respectively, for $\left(Q_{1 c}, Q_{d}, Q_{2 c}\right)=\left(150 \mu 1 \mathrm{~h}^{-1}\right.$, $\left.30 \mu 1 \mathrm{~h}^{-1}, 50 \mu \mathrm{h}^{-1}\right)$. The analytical expression of the pressure waveform is obtained by using a curve fitting tool (sum of sines model with two terms) in MATLAB (Mathworks, USA) and expressed as

$$
\Delta p^{*}=11.67 \sin (11.33 t *+2.683)+24.67 \sin (0.0333 t *+2.794) .
$$

The units of $\left(\Delta p^{*}\right)$ and $t^{*}$ in (3.2) are Pascals $(\mathrm{Pa})$ and seconds (s), respectively. The first term (high-frequency component) of (3.2) makes the frequency of the differential pressure waveform consistent with the experimental results, whereas the addition of the second term (very low-frequency component) renders the amplitude consistent.

Figures $16(a)$ and $16(b)$ illustrate a comparison between the numerically and experimentally obtained droplet shapes and migration characteristics, respectively, in the combined presence of an axial electric field and background oscillatory flow. First of all, 
(a)

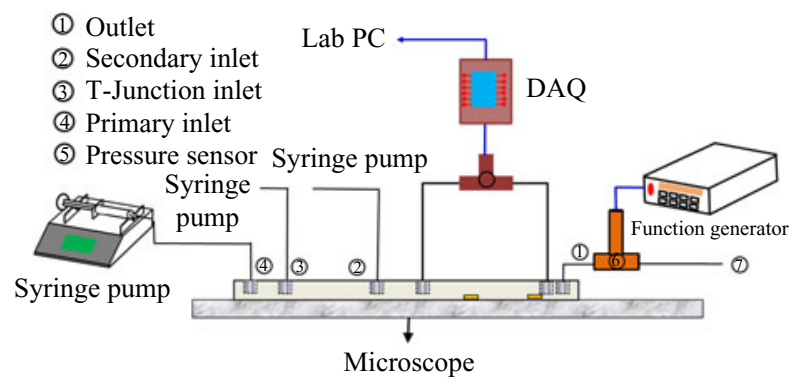

(b)
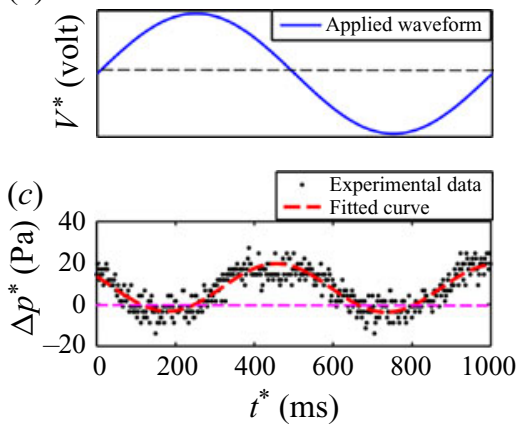

FIGURE 15. (a) Schematic illustration of the experimental set-up for measuring the pressure differential across the channel length, $(b)$ applied voltage waveform and $(c)$ obtained differential pressure waveform.

(a)

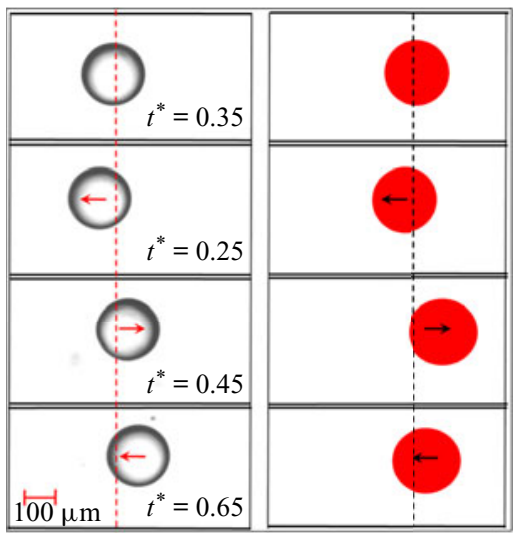

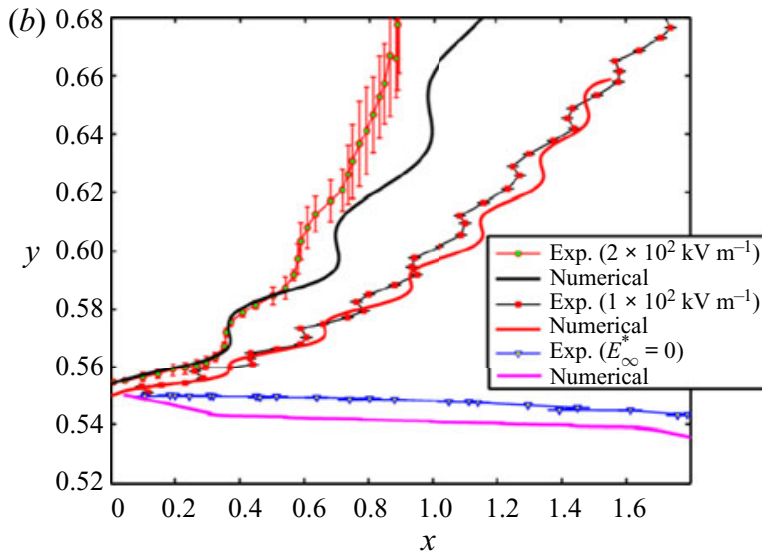

FIgURE 16. (a) Comparison between experimentally and numerically obtained droplet configurations, at $E_{\infty}^{*}=1 \times 10^{2} \mathrm{kV} \mathrm{m}^{-1}$. (b) Comparison between experimentally and numerically obtained migration characteristics. Other parameters are $C a=0.0015$, $(R, S)=\left(O\left(10^{4}\right), 20.1\right), \lambda=0.05, W c=0.47$ and $R e \sim 10^{-2}$.

these figures show that the axial electric field has a profound effect in confined domain unlike for the case of unbounded domain (Mandal et al. 2016). Second, figure 16(b) points out that for system $\mathrm{B}$, having $R>S$, the higher strength of the electric field not only suppresses the axial oscillation of the droplet in its cross-stream motion, but also drives to droplet to the wall nearest to it at a faster rate. These figures also demonstrate that the numerically and experimentally obtained droplet shapes and migration characteristics exhibit good agreement and justify the validity of the numerical findings.

Next, we experimentally demonstrate the effect of the electric field on the cross-stream migration characteristics of the droplet for system A, having $S>R$, as depicted in figure 17 . For the present system, the values of $Q_{d}, Q_{1 c}$ and $Q_{2 c}$ are taken as $20 \mu \mathrm{lh}^{-1}, 130 \mu 1 \mathrm{~h}^{-1}$ and $35 \mu 1 \mathrm{~h}^{-1}$, respectively, and the approximate analytical expression of the differential waveform is expressed as

$$
\Delta p^{*}=11.81 \sin (11.33 t *+2.847)+26.36 \sin (0.03937 t *+2.974) \text {. }
$$




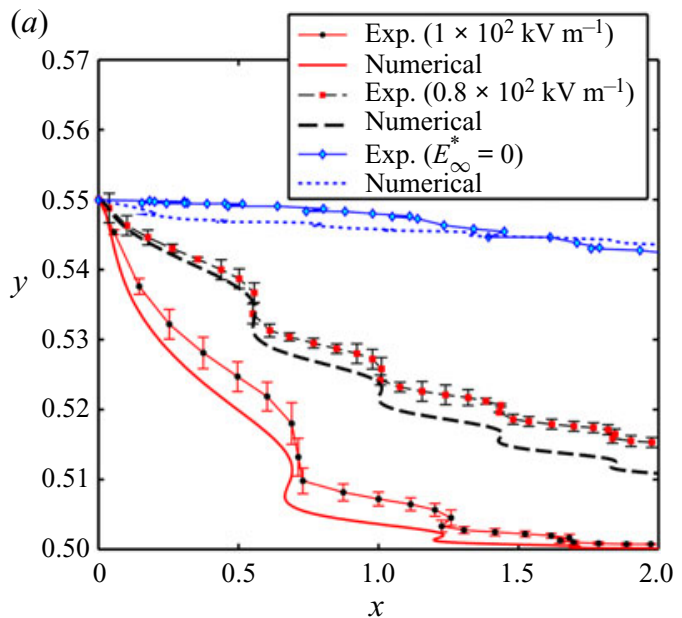

(i)

(b)

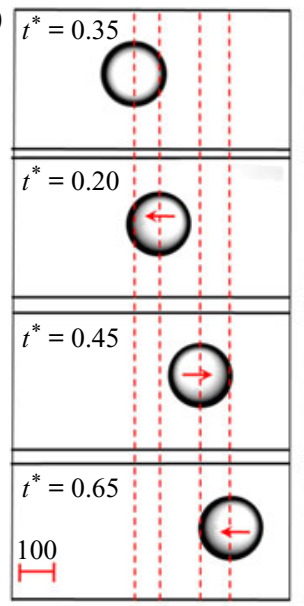

(ii)

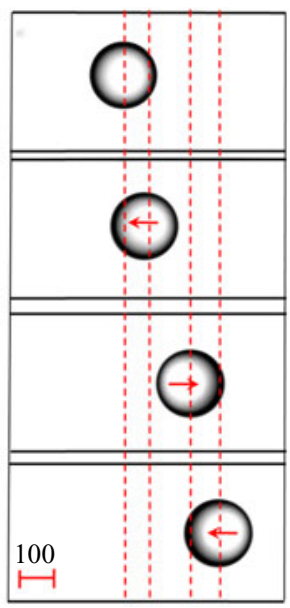

FIGURE 17. (a) Effect of electric field strength on the cross-stream motion of the droplets. (b) Shape and position of the droplet at different times for (i) $E_{\infty}^{*}=0$ and (ii) $0.8 \times 10^{2} \mathrm{kV} \mathrm{m}^{-1}$. Other parameters are $(R, S)=(0.02,1.19), \lambda=0.42, C a=0.006, W c=0.45$ and $R e \sim 10^{-2}$.

From figure 17(a), we observe that as we increase the strength of the electric field, the axial oscillation of the droplets prior to reaching the centreline attenuates and the droplet reaches the centreline at a faster rate, justifying the theoretical findings. In figure 17(b), we demonstrate the experimentally obtained shapes and positions of the droplet at different times for different values of the electric field, as the primary observable characteristic governing the migration dynamics.

Next, we experimentally demonstrate the domain confinement-induced alteration in cross-stream migration of the droplet in system A, under the combined presence of a steady axial electric field and confined oscillatory microflow, as shown in figure 18. To vary the confinement ratio as mediated by the size of the droplet, we have varied the flow rate of the dispersed fluid $\left(Q_{d}\right)$, to enable alterations in the initial droplet size. Figure 18( $\left.a\right)$ shows that the axial oscillations of the droplet before reaching the centreline dampen out with the increase in the value of $W c$ (degree of confinement) and the droplet migrates towards the centreline at a faster rate. This corroborates our theoretical observations. For $W c=0.72$, for instance, the droplet reaches the centreline without undergoing any axial oscillations. Physical reasoning behind such observed phenomenon has been presented in the discussions on our theoretical findings. In figure $18(b)$, we further depict the experimentally obtained shape and position of the droplet at different time instances, for different confinement ratios. Such deformation characteristics are essentially responsible for the observed interplay between the electrical and hydrodynamic influences mediated by confinement-induced interactions. These results of fundamental importance towards developing extended constitutive forms and physical models of transport phenomena observed in nature and engineering (Ganguly \& Chakraborty 2004; Pal et al. 2006; Chakraborty \& Durst 2007; Chakraborty \& Padhy 2008; Dongari, Durst \& Chakraborty 2010; Kar, Maiti \& Chakraborty 2015; Bandopadhyay et al. 2016; Poddar et al. 2016; Kunti, Bhattacharya \& Chakraborty 2017). 
(i)

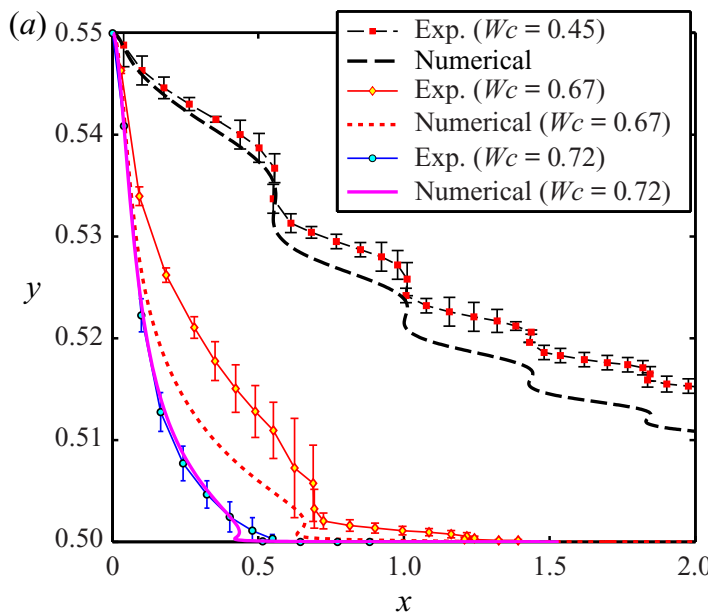

(b)

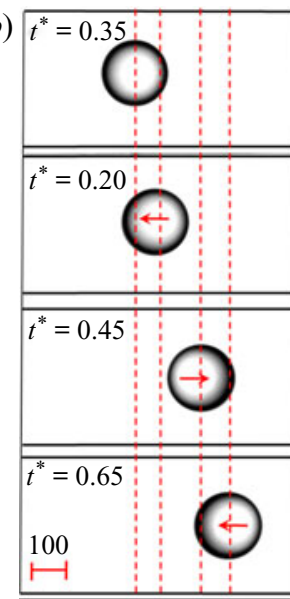

(ii)

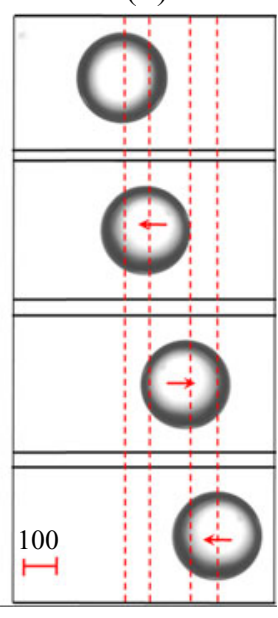

FIGURE 18. (a) Effect of domain confinement on the cross-stream motion of the droplets at $E_{\infty}^{*}=0.8 \times 10^{2} \mathrm{kV} \mathrm{m}^{-1}$. (b) Shape and position of the droplet at different times for (i) $W c=0.45$ and (ii) $W c=0.72$. Other parameters are $(R, S)=(0.02,1.19), \lambda=0.42, C a=0.006$ and $R e \sim 10^{-2}$.

\section{Conclusions}

We have predicted theoretically and confirmed via experimental studies that the combined effect of confinement, electromechanics and hydrodynamics gives rise to several artifacts in the cross-stream motion of a droplet in the combined presence of an axial electric field and oscillatory microflow, which are non-existent in an unbounded steady flow under axial electrical forcing. Important findings from our study are as follows.

(i) For leaky dielectric systems having $S>R$ and $\Omega_{T}<0$, the presence of an axial electric field facilitates rapid cross-stream migration of the droplet via suppressing the axial oscillations, prior to reaching the channel centreline. Complete attenuation of the oscillatory characteristics of the droplet migration takes place beyond a threshold value of $C a_{E}$ that increases with the rise in the values of St.

(ii) For leaky dielectric systems having $R>S$ and $\Omega_{T}>0$, at moderate values of $C a_{E}$, the presence of an electric field induces additional axial oscillations in the migration characteristics and delays the cross-stream motion of the droplet towards the centreline. For a slight increase in the magnitude of $C a_{E}$, the droplet achieves the steady-state transverse position almost instantaneously, where the initial and final positions of the droplet are virtually identical. In contrast, beyond a threshold value of $C a_{E}$, a simultaneous reversal of the droplet motion and an enhancement of the rate of cross-stream migration of the droplet can be noted.

(iii) Domain confinement is also found to have a significant effect in suppressing axial oscillations in the cross-stream motion of the droplet as well as in the conversion of its migration pattern from oscillatory to oscillation-free. With a rise in the degree of confinement, the time taken by the droplet to attain a steady-state transverse position also decreases.

(iv) The electrical property ratio $(R / S)$ also shows a profound effect on the cross-stream migration. Below a threshold value of $R / S$, the migration rate of the droplet is significantly high and the time taken by the droplet to reach a steady-state transverse 
position is low. Depending on the values of $R$ and $S$, the droplet exhibits three distinct patterns of migration: (a) oscillatory motion towards the adjacent wall, (b) oscillatory motion towards the channel centreline and (c) oscillation-free motion towards the channel centreline.

The important findings listed here implicate that the interplay between the electromechanics and the oscillatory hydrodynamics in a confined fluidic environment may potentially be translated into a physics-based unified design principle, holding the potential of becoming the fundamental premise of developing a versatile and efficient in vitro platform for performing several processes of emerging impact, innovation and interest encompassing a wide variety of applications ranging from engineering to biology.

\section{Acknowledgements}

S.S. is grateful to Dr S. Mandal and Dr S. Das for insightful discussions on droplet electrohydrodynamics. S.S. is thankful to Mr S. Bandyopadhyay for the insightful discussion on the process of differential pressure measurement. S.C. acknowledges the Department of Science and Technology, Government of India, for the Sir J.C. Bose National Fellowship.

\section{Declaration of interests}

The authors declare no competing interest.

\section{Supplementary material}

The supplementary material consists of the details of the numerical simulation, grid independence study and Cahn number independence study.

Supplementary material are available at https://doi.org/10.1017/jfm.2020.789.

\section{REFERENCES}

AfKhami, S., Leshansky, A. M. \& Renardy, Y. 2011 Numerical investigation of elongated drops in a microfluidic T-junction. Phys. Fluids 23 (2), 022002.

Asghari, M., Cao, X., Mateescu, B., Van leeunen, D., Aslan, M. K., Stavrakis, S. \& DeMello, A. J. 2020 Oscillatory viscoelastic microfluidics for efficient focusing and separation of nanoscale species. ACS Nano 14 (1), 422-433.

Badalassi, V. E., Ceniceros, H. D. \& BanerJee, S. 2003 Computation of multiphase systems with phase field models. J. Comput. Phys. 190 (2), 371-397.

BAKLI, C. \& ChaKRaborty, S. 2012 Capillary filling dynamics of water in nanopores. Appl. Phys. Lett. 101 (15), 153112.

BANDOPADHYAY, A. \& CHAKRABORTY, S. 2012a Combined effects of interfacial permittivity variations and finite ionic sizes on streaming potentials in nanochannels. Langmuir 28 (50), 17552-17563.

BANDOPADHYAY, A. \& CHAKRABORTY, S. $2012 b$ Electrokinetically induced alterations in dynamic response of viscoelastic fluids in narrow confinements. Phys. Rev. E 85 (5), 056302.

Bandopadhyay, A., Mandal, S., Kishore, N. K. \& ChaKraborty, S. 2016 Uniform electric-field-induced lateral migration of a sedimenting drop. J. Fluid Mech. 792 (May), 553-589.

Behera, N., MANDAL, S. \& ChaKraborty, S. 2019 Electrohydrodynamic settling of drop in uniform electric field: beyond Stokes flow regime. J. Fluid Mech. 881, 498-523.

Brosseau, Q. \& VlahovsKa, P. M. 2017 Streaming from the equator of a drop in an external electric field. Phys. Rev. Lett. 119 (3), 034501. 
Capretto, L., Cheng, W., Hill, M. \& Zhang, X. 2011 Micromixing within microfluidic devices. In Microfluidics. Topics in Current Chemistry, vol. 304, pp. 27-68. Springer.

CArlson, A., Do-Quang, M. \& Amberg, G. 2010 Droplet dynamics in a bifurcating channel. Intl J. Multiphase Flow 36 (5), 397-405.

Casadevall i Solvas, X. \& DeMello, A. 2011 Droplet microfluidics: recent developments and future applications. Chem. Commun. 47 (7), 1936-1942.

Chaffey, C. E., Brenner, H. \& Mason, S. G. 1965 Particle motions in sheared suspensions. Rheol. Acta 4 (1), 64-72.

CHAKRABORTY, S. 2007 Order parameter modeling of fluid dynamics in narrow confinements subjected to hydrophobic interactions. Phys. Rev. Lett. 99 (9), 094504.

CHAKRABORTY, S. 2008 Order parameter description of electrochemical-hydrodynamic interactions in nanochannels. Phys. Rev. Lett. 101 (18), 184501.

Chakraborty, S. \& DURST, F. 2007 Derivations of extended Navier-Stokes equations from upscaled molecular transport considerations for compressible ideal gas flows: towards extended constitutive forms. Phys. Fluids 19 (8), 88104.

Chakraborty, S. \& PADHY, S. 2008 Anomalous electrical conductivity of nanoscale colloidal suspensions. ACS Nano 2 (10), 2029-2036.

Chan, P. C.-H. \& LeAL, L. G. 1977 A note on the motion of a spherical particle in a general quadratic flow of a second-order fluid. J. Fluid Mech. 82 (3), 549-559.

Chan, P. C.-H. \& LEAL, L. G. 1979 The motion of a deformable drop in a second-order fluid. J. Fluid Mech. 92 (1), 131-170.

Chaudhury, K., Mandal, S. \& Chakraborty, S. 2016 Droplet migration characteristics in confined oscillatory microflows. Phys. Rev. E 93 (2), 023106.

Chen, X., Xue, C., Zhang, L., Hu, G., JiAng, X. \& Sun, J. 2014 Inertial migration of deformable droplets in a microchannel. Phys. Fluids 26 (11), 112003.

Chung, C., Lee, M., Char, K., Ahn, K. H. \& Lee, S. J. 2010 Droplet dynamics passing through obstructions in confined microchannel flow. Microfluid Nanofluid 9 (6), 1151-1163.

Dangla, R., KaYi, S. C. \& BARoud, C. N. 2013 Droplet microfluidics driven by gradients of confinement. Proc. Natl. Acad. Sci. 110 (3), 853-858.

Deshmukh, S. D. \& Thaokar, R. M. 2012 Deformation, breakup and motion of a perfect dielectric drop in a quadrupole electric field. Phys. Fluids 24 (3), 032105.

Dey, R., Chakraborty, D. \& Chakraborty, S. 2011 Analytical solution for thermally fully developed combined electroosmotic and pressure-driven flows in narrow confinements with thick electrical double layers. Trans. ASME: J. Heat Transfer 133 (2), 24503.

Dey, R., Shaik, V. A., Chakraborty, D., Ghosal, S. \& Chakraborty, S. 2015 AC electric field-induced trapping of microparticles in pinched microconfinements. Langmuir 31 (21), 5952-5961.

Dongari, N., Durst, F. \& Chakraborty, S. 2010 Predicting microscale gas flows and rarefaction effects through extended Navier-Stokes-Fourier equations from phoretic transport considerations. Microfluid Nanofluid 9 (4-5), 831-846.

ESMAEELI, A. 2016 Dielectrophoretic- and electrohydrodynamic-driven translational motion of a liquid column in transverse electric fields. Phys. Fluids 28 (7), 073306.

GANGUly, S. \& CHAKRABorty, S. 2004 Numerical investigation on role of bottom gas stirring in controlling thermal stratification in steel ladles. ISIJ Intl 44 (3), 537-546.

Goldsmith, H. \& MASON, S. 1962 The flow of suspensions through tubes. I. Single spheres, rods, and discs. J. Colloid Sci. 17 (5), 448-476.

Goswami, P. \& Chakraborty, S. 2011 Semi-analytical solutions for electroosmotic flows with interfacial slip in microchannels of complex cross-sectional shapes. Microfluid Nanofluid 11 (3), 255-267.

GRAhAM, D. R. \& Higdon, J. J. L. $2000 a$ Oscillatory flow of droplets in capillary tubes. Part 1. Straight tubes. J. Fluid Mech. 425, 31-53.

Graham, D. R. \& Higdon, J. J. L. $2000 b$ Oscillatory flow of droplets in capillary tubes. Part 2. Constricted tubes. J. Fluid Mech. 425, 55-77.

Graham, D. R. \& Higdon, J. J. L. 2002 Oscillatory forcing of flow through porous media. Part 1. Steady flow. J. Fluid Mech. 465, 213-235. 
HA, J.-W. \& YANG, S. 2000 Deformation and breakup of Newtonian and non-Newtonian conducting drops in an electric field. J. Fluid Mech. 405, 131-156.

Haber, S. \& Hetsroni, G. 1971 The dynamics of a deformable drop suspended in an unbounded Stokes flow. J. Fluid Mech. 49 (2), 257-277.

Halim, M. A. \& Esmaeeli, A. 2013 Computational studies on the transient electrohydrodynamics of a liquid drop. Fluid Dyn. Mater. Process. 9 (4), 435-460.

Hase, M., Watanabe, S. N. \& Yoshikawa, K. 2006 Rhythmic motion of a droplet under a dc electric field. Phys. Rev. E 74 (4), 046301.

Hazra, S., Mitra, S. K. \& SEN, A. K. 2019 Lateral migration of viscoelastic droplets in a viscoelastic confined flow: role of discrete phase viscoelasticity. Soft Matter 15 (44), 9003-9010.

Ho, B. P. \& LEAL, L. G. 1974 Inertial migration of rigid spheres in two-dimensional unidirectional flows. J. Fluid Mech. 65 (2), 365-400.

Huerre, A., Theodoly, O., Leshansky, A. M., Valignat, M.-P., Cantat, I. \& Jullien, M.-C. 2015 Droplets in microchannels: dynamical properties of the lubrication film. Phys. Rev. Lett. 115 (6), 064501.

JACQMIN, D. 1999 Calculation of two-phase Navier-Stokes flows using phase-field modeling. J. Comput. Phys. 155 (1), 96-127.

Kar, S., Maiti, T. K. \& Chakraborty, S. 2015 Capillarity-driven blood plasma separation on paper-based devices. Analyst 140 (19), 6473-6476.

Kunti, G., Bhattacharya, A. \& ChaKraborty, S. 2017 Rapid mixing with high-throughput in a semi-active semi-passive micromixer. Electrophoresis 38 (9-10), 1310-1317.

LEAL, L. G. 1980 Particle motions in a viscous fluid. Annu. Rev. Fluid Mech. 12 (1), 435-476.

LI, X. \& PozRiKidis, C. 2002 Film flow of a suspension of liquid drops. Phys. Fluids 14 (1), 61-74.

Lovalenti, P. M. \& BRADY, J. F. 1993 The force on a bubble, drop, or particle in arbitrary time-dependent motion at small Reynolds number. Phys. Fluids A Fluid Dyn. 5 (9), 2104-2116.

Mandal, S., Bandopadhyay, A. \& Chakraborty, S. 2015a Effect of interfacial slip on the cross-stream migration of a drop in an unbounded Poiseuille flow. Phys. Rev. E 92 (2), 023002.

MANDAL, S., BANDOPAdHYAy, A. \& CHAKRABORTY, S. 2016 The effect of uniform electric field on the cross-stream migration of a drop in plane Poiseuille flow. J. Fluid Mech. 809, 726-774.

Mandal, S., Ghosh, U., Bandopadhyay, A. \& Chakraborty, S. 2015b Electro-osmosis of superimposed fluids in the presence of modulated charged surfaces in narrow confinements. J. Fluid Mech. 776, 390-429.

MELCHER, J. R. \& TAYLOR, G. I. 1969 Electrohydrodynamics: a review of the role of interfacial shear stresses. Annu. Rev. Fluid Mech. 1, 111-146.

Mhatre, S. \& THAOKAR, R. M. 2013 Drop motion, deformation, and cyclic motion in a non-uniform electric field in the viscous limit. Phys. Fluids 25 (7), 072105.

Mondal, P. K., Ghosh, U., Bandopadhyay, A., DasGupta, D. \& Chakraborty, S. 2014 Pulsating electric field modulated contact line dynamics of immiscible binary systems in narrow confinements under an electrical double layer phenomenon. Soft Matter 10 (42), 8512-8523.

Mortazavi, S. \& Tryggvason, G. 2000 A numerical study of the motion of drops in Poiseuille flow. Part 1. Lateral migration of one drop. J. Fluid Mech. 411, 325-350.

MUKHERJEe, S. \& SARKAR, K. 2013 Effects of matrix viscoelasticity on the lateral migration of a deformable drop in a wall-bounded shear. J. Fluid Mech. 727, 318-345.

MUKHERJEe, S. \& SARKAR, K. 2014 Lateral migration of a viscoelastic drop in a Newtonian fluid in a shear flow near a wall. Phys. Fluids 26 (10), 103102.

Mutlu, B. R., EdD, J. F. \& TONER, M. 2018 Oscillatory inertial focusing in infinite microchannels. Proc. Natl Acad. Sci. 115 (30), 7682-7687.

NAth, B., Biswas, G., Dalal, A. \& SAHU, K. C. 2018 Cross-stream migration of drops suspended in Poiseuille flow in the presence of an electric field. Phys. Rev. E 97 (6), 063106.

Pal, D., Bhattacharya, J., Dutta, P. \& Chakraborty, S. 2006 An enthalpy model for simulation of dendritic growth. Numer. Heat Transfer B Fundam. 50 (1), 59-78.

Peters, F. \& ARABALI, D. 2013 Interfacial tension between oil and water measured with a modified contour method. Colloids Surf. A Physicochem. Engng Asp. 426, 1-5. 
Poddar, A., Maity, D., Bandopadhyay, A. \& Chakraborty, S. 2016 Electrokinetics in polyelectrolyte grafted nanofluidic channels modulated by the ion partitioning effect. Soft Matter 12 (27), 5968-5978.

Poddar, A., Mandal, S., Bandopadhyay, A. \& Chakraborty, S. 2018 Sedimentation of a surfactant-laden drop under the influence of an electric field. J. Fluid Mech. 849, $277-311$.

Poddar, A., Mandal, S., BAndopadhyay, A. \& ChaKraborty, S. $2019 a$ Electrical switching of a surfactant coated drop in Poiseuille flow. J. Fluid Mech. 870, 27-66.

Poddar, A., Mandal, S., Bandopadhyay, A. \& Chakraborty, S. $2019 b$ Electrorheology of a dilute emulsion of surfactant-covered drops. J. Fluid Mech. 881, 524-550.

Rana, U., Chakraborty, S. \& Som, S. K. 2014 Thermodynamics of premixed combustion in a heat recirculating micro combustor. Energy $68,510-518$.

Ristenpart, W. D., Bird, J. C., Belmonte, A., Dollar, F. \& Stone, H. A. 2009 Non-coalescence of oppositely charged drops. Nature 461 (7262), 377-380.

SANTRA, S., DAS, S. \& CHAKRABORTY, S. 2020 Electrically modulated dynamics of a compound droplet in a confined microfluidic environment. J. Fluid Mech. 882, A23.

SAntra, S., DAS, S., DAS, S. S. \& Chakraborty, S. 2018a Surfactant-induced retardation in lateral migration of droplets in a microfluidic confinement. Microfluid Nanofluid 22 (8), 88.

SAntra, S., MANDAL, S. \& ChAKRABORTY, S. $2018 b$ Electrohydrodynamics of confined two-dimensional liquid droplets in uniform electric field. Phys. Fluids 30 (6), 062003.

Santra, S., Mandal, S. \& Chakraborty, S. 2019a Confinement effect on electrically induced dynamics of a droplet in shear flow. Phys. Rev. E 100 (3), 033101.

SAntra, S., Sen, D., DAS, S. \& Chakraborty, S. $2019 \mathrm{~b}$ Electrohydrodynamic interaction between droplet pairs in a confined shear flow. Phys. Fluids 31 (3), 032005.

SARKAR, K. \& SCHOWALTER, W. R. 2001 $a$ Deformation of a two-dimensional drop at non-zero Reynolds number in time-periodic extensional flows: numerical simulation. J. Fluid Mech. 436, 177-206.

SARKAR, K. \& Schowalter, W. R. $2001 b$ Deformation of a two-dimensional viscous drop in time-periodic extensional flows: analytical treatment. J. Fluid Mech. 436, 207-230.

SAVILLE, D. A. 1997 Electrohydrodynamics: the Taylor-Melcher leaky dielectric model. Annu. Rev. Fluid Mech. 29 (1), 27-64.

Schwabe, D., Möller, U., Schneider, J. \& Scharmann, A. 1992 Instabilities of shallow dynamic thermocapillary liquid layers. Phys. Fluids A Fluid Dyn. 4 (11), 2368-2381.

SeČ, D., Porenta, T., Ravnik, M. \& ŽUmer, S. 2012 Geometrical frustration of chiral ordering in cholesteric droplets. Soft Matter 8 (48), 11982.

Sessoms, D. A., Belloul, M., Engl, W., Roche, M., Courbin, L. \& Panizza, P. 2009 Droplet motion in microfluidic networks: Hydrodynamic interactions and pressure-drop measurements. Phys. Rev. E 80 (1), 016317.

Sibillo, V., Pasquariello, G., Simeone, M., Cristini, V. \& Guido, S. 2006 Drop deformation in microconfined shear flow. Phys. Rev. Lett. 97, 054502.

Stan, C. A., Guglielmini, L., Ellerbee, A. K., Caviezel, D., Stone, H. A. \& Whitesides, G. M. 2011 Sheathless hydrodynamic positioning of buoyant drops and bubbles inside microchannels. Phys. Rev. E 84 (3), 036302.

Stone, H. A., Stroock, A. D. \& AJdari, A. 2004 Engineering flows in small devices: microfluidics toward a Lab-on-a-Chip. Annu. Rev. Fluid Mech. 36 (1), 381-411.

TAYLOR, G. 1966 Studies in electrohydrodynamics. I. The circulation produced in a drop by an electrical field. Proc. R. Soc. A 291 (1425), 159-166.

TEH, S.-Y., Lin, R., Hung, L.-H. \& LeE, A. P. 2008 Droplet microfluidics. Lab on a Chip 8 (2), 198.

Torza, S., Cox, R. G. \& MAson, S. G. 1971 Electrohydrodynamic deformation and burst of liquid drops. Philos. Trans. R. Soc. A Math. Phys. Engng Sci. 269 (1198), 295-319.

ViziKa, O. \& SAVILLE, D. A. 1992 The electrohydrodynamic deformation of drops suspended in liquids in steady and oscillatory electric field. J. Fluid Mech. 239, 1-21.

Wang, Q., Jones, A.-A. D., Gralnick, J. A., Lin, L. \& Buie, C. R. 2019 Microfluidic dielectrophoresis illuminates the relationship between microbial cell envelope polarizability and electrochemical activity. Sci. Adv. 5 (1), 1-11.

WANG, X.-P., QIAN, T. \& SHENG, P. 2008 Moving contact line on chemically patterned surfaces. J. Fluid Mech. 605, 59-78. 
Wioland, H., Woodhouse, F. G., Dunkel, J., Kessler, J. O. \& Goldstein, R. E. 2013 Confinement stabilizes a bacterial suspension into a spiral vortex. Phys. Rev. Lett. 110 (26), 268102.

Wohl, P. R. \& Rubinow, S. I. 1974 The transverse force on a drop in an unbounded parabolic flow. J. Fluid Mech. 62 (1), 185-207.

YANG, Q., LI, B. Q. \& DING, Y. 2013 3D phase field modeling of electrohydrodynamic multiphase flows. Intl J. Multiph. Flow 57, 1-9.

YAng, Q., Li, B. Q., ZhaO, Z., ShaO, J. \& XU, F. 2016 Numerical analysis of the Rayleigh-Taylor instability in an electric field. J. Fluid Mech. 792, 397-434.

Yavari, H., Sadeghi, A., Saidi, M. H. \& Chakraborty, S. 2012 Combined influences of viscous dissipation, non-uniform Joule heating and variable thermophysical properties on convective heat transfer in microtubes. Intl J. Heat Mass Transfer 55 (4), 762-772.

Zhang, Y., Zhu, B., LiU, Y. \& Wittstock, G. 2016 Hydrodynamic dispensing and electrical manipulation of attolitre droplets. Nat. Commun. 7, 12424. 